\title{
Spectral study of the diffuse synchrotron source in the galaxy cluster Abell 523
}

Valentina Vacca, ${ }^{1 \star}$, Timothy Shimwell ${ }^{2}$, Richard A. Perley ${ }^{3}$, Federica Govoni ${ }^{1}$, Matteo Murgia ${ }^{1}$, Luigina Feretti ${ }^{4}$, Gabriele Giovannini ${ }^{4,5}$, Francesca Loi ${ }^{1}$, Ettore Carretti ${ }^{4}$, Filippo Cova ${ }^{6}$, Fabio Gastaldello $^{6}$, Marisa Girardi ${ }^{8}$, Torsten Enßlin ${ }^{7}$, Hiroki Akamatsu ${ }^{9}$, Annalisa Bonafede ${ }^{4,5}$, Etienne Bonnassieux ${ }^{5}$, Walter Boschin ${ }^{10,11,12}$, Andrea Botteon ${ }^{9}$, Gianfranco Brunetti ${ }^{4}$, Marcus Brüggen ${ }^{13}$, Alexis Finoguenov ${ }^{14}$, Duy Hoang ${ }^{13}$, Marco Iacobelli ${ }^{2}$, Emanuela Orrú ${ }^{2}$, Rosita Paladino ${ }^{4}$, Huub Röttgering ${ }^{9}$, Reinout van Weeren ${ }^{9}$, Fabio Vitello ${ }^{4}$, Denis Wittor ${ }^{13,5}$

${ }^{1}$ INAF-Osservatorio Astronomico di Cagliari, Via della Scienza 5, I-09047 Selargius (CA), Italy

${ }^{2}$ ASTRON, the Netherlands Institute for Radio Astronomy, Postbus 2, 7990 AA, Dwingeloo, The Netherlands

${ }^{3}$ National Radio Astronomy Observatory, P.O.Box O, Socorro, NM, 87801

${ }^{4}$ INAF - Istituto di Radioastronomia, Via P. Gobetti 101, 40129 Bologna, Italy

${ }^{5}$ Dipartimento di Fisica e Astronomia, Universita di Bologna, Via Gobetti 93/2, 40122, Bologna, Italy

${ }^{6}$ IASF - Milano, INAF, Via Corti 12, I-20133 Milan, Italy

${ }^{7}$ Max Planck Institute for Astrophysics, Karl-Schwarzschildstr. 1, 85741 Garching, Germany

${ }^{8}$ Dipartimento di Fisica dell'Università degli Studi di Trieste - Sezione di Astronomia, via Tiepolo 11, I-34143 Trieste, Italy

${ }^{9}$ Leiden Observatory, Leiden University, P.O. Box 9513, 2300 RA Leiden, The Netherlands

${ }^{10}$ Fundación G. Galilei - INAF (Telescopio Nazionale Galileo), Rambla J. A. Fernández Pérez 7, E-38712 Breña Baja (La Palma), Spain

${ }^{11}$ Instituto de Astrofísica de Canarias, C/Vía Lactea s/n, E-38205 La Laguna (Tenerife), Spain

${ }^{12}$ Departamento de Astrofísica, Univ. de La Laguna, Av. del Astrofísico Francisco Sánchez, s/n, E-38205 La Laguna (Tenerife), Spain

${ }^{13}$ University of Hamburg, Gojenbergsweg 112, 21029 Hamburg, Germany

${ }^{14}$ Department of Physics, University of Helsinki, Gustaf Hällströmin katu 2, FI-00014 Helsinki, Finland

Accepted for publication on MNRAS

\begin{abstract}
The galaxy cluster Abell 523 (A523) hosts an extended diffuse synchrotron source historically classified as a radio halo. Its radio power at $1.4 \mathrm{GHz}$ makes it one of the most significant outliers in the scaling relations between observables derived from multi-wavelength observations of galaxy clusters: it has a morphology that is different and offset from the thermal gas, and it has polarized emission at $1.4 \mathrm{GHz}$ typically difficult to observe for this class of sources. A magnetic field fluctuating on large spatial scales $(\sim 1 \mathrm{Mpc})$ can explain these peculiarities but the formation mechanism for this source is not yet completely clear. To investigate its formation mechanism, we present new observations obtained with the LOw Frequency ARray at 120-168 MHz and the Jansky Very Large Array at 1-2 GHz, which allow us to study the spectral index distribution of this source. According to our data the source is observed to be more extended at $144 \mathrm{MHz}$ than previously inferred at $1.4 \mathrm{GHz}$, with a total size of about $1.8 \mathrm{Mpc}$ and a flux density $S_{144 \mathrm{MHz}}=(1.52 \pm 0.31) \mathrm{Jy}$. The spectral index distribution of the source is patchy with an average spectral index $\alpha \sim 1.2$ between $144 \mathrm{MHz}$ and $1.410 \mathrm{GHz}$, while an integrated spectral index $\alpha \sim 2.1$ has been obtained between $1.410 \mathrm{GHz}$ and $1.782 \mathrm{GHz}$. A previously unseen patch of steep spectrum emission is clearly detected at $144 \mathrm{MHz}$ in the south of the cluster. Overall, our findings suggest that we are observing an overlapping of different structures, powered by the turbulence associated with the primary and a possible secondary merger.
\end{abstract}

Key words: acceleration of particles- magnetic fields- galaxies: clusters: intracluster medium- cosmology: observationslarge-scale structure of Universe

\section{INTRODUCTION}

Diffuse synchrotron sources observed in merging galaxy clusters indicate the presence of ultra-relativistic electrons $(\gamma>>1000)$ spiralling along the flux lines of weak magnetic fields $(\sim \mu \mathrm{G})$, and

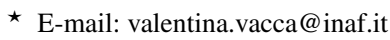

can be classified as (giant) halos and relics (e.g., Feretti et al. 2012; Brunetti \& Jones 2014; Vacca et al. 2018a; van Weeren et al. 2019).

Radio halos have been observed in a fraction of massive and disturbed galaxy clusters where they fill the central volume of the system (Cassano et al. 2010). The prototype and most famous radio halo is Coma $\mathrm{C}$ which resides in the Coma cluster and was first observed by Large et al. (1959). Overall, radio halos extend up to spatial 
scales of $\sim 1-2 \mathrm{Mpc}$ and are characterized by low radio brightness $\left(\sim 0.1 \mu \mathrm{Jy} / \operatorname{arcsec}^{2}\right.$ at $\left.1.4 \mathrm{GHz}\right)$. Out of about 100 radio halos presently confirmed, three show filaments of polarized emission at around $20 \%$ at $1.4 \mathrm{GHz}$ (A2255 Govoni et al. 2005, MACS J0717.5+3745 Bonafede et al. 2009, and A523 Girardi et al. 2016). These are likely projected on the cluster center as suggested by recent studies of A2255 and MACS J0717.5+3745 (Pizzo et al. 2011; Botteon et al. 2020; Rajpurohit et al. 2020). Moreover, a $5 \sigma$ upper limit of $13 \%$ on the fractional polarization has been derived for the radio halo in 1E 0657-55.8 by Shimwell et al. (2014). Three-dimensional numerical simulations suggest that radio halos are intrinsically polarized at $1.4 \mathrm{GHz}$, with filaments of polarized emission expected to be observed at distances greater than $1.5 \mathrm{Mpc}$ from the cluster center, reflecting the intracluster magnetic field structure (Loi et al. 2019). However, the resolution and sensitivity of present instruments hinder the detection of this polarized emission in most radio halos (Govoni et al. 2013).

Typically, most powerful and extended radio halos are hosted in massive and bright X-ray clusters and show strong correlation between the integrated radio halo emission at $1.4 \mathrm{GHz}$ and the X-ray luminosity of the intracluster medium (ICM) and galaxy cluster mass (e.g., Liang et al. 2000; Cassano et al. 2013; Yuan et al. 2015; Cuciti et al. 2021). Exceptions have been observed of radio halos that show a radio power at $1.4 \mathrm{GHz}$ larger than expected from the radio power

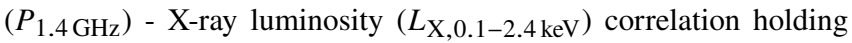
for the most of radio halos, one of the most significant being the source in A523 (Giovannini et al. 2011) ${ }^{1}$. Detailed studies indicate, in some cases, a point-to-point correlation between the X-ray and radio brightness at $1.4 \mathrm{GHz}$ as, e.g., for the radio halos in the Coma cluster and 1RXS J0603.3+4214 (Govoni et al. 2001a; Brown \& Rudnick 2011; Rajpurohit et al. 2018), while a minor (e.g., A520 Govoni et al. 2001b; Hoang et al. 2019) or absent (e.g., 1E 0657-55.8 Shimwell et al. 2014) correlation has been found in other clusters.

Radio relics are elongated diffuse synchrotron sources with filamentary morphology that are observed in the periphery of a number of merging galaxy clusters. Sometimes they come in pairs along the merger axis but on opposite sides (e.g., Rottgering et al. 1997; Bagchi et al. 2006; van Weeren et al. 2011) and/or coexist with cluster radio halos (e.g., Brown et al. 2011; Lindner et al. 2014; Parekh et al. 2017). They are extended on spatial scales of $\sim 0.5-2 \mathrm{Mpc}$, are characterized by low radio surface brightness $\left(\sim 0.1 \mu \mathrm{Jy} / \operatorname{arcsec}^{2}\right.$ at $\left.1.4 \mathrm{GHz}\right)$, and are highly polarized at $\mathrm{GHz}$ frequencies with fractional polarization larger than $20 \%$ at $1.4 \mathrm{GHz}$.

In our present understanding, a key role in the origin of radio halos and relics is likely played by cluster merger phenomena in the form of turbulence and shocks. Merger-induced turbulence is thought to accelerate a pre-existing electron population (seed population) through the Fermi-II mechanism, giving rise to diffuse synchrotron sources filling the central volume, i.e. radio halos, of a fraction of massive galaxy clusters. According to this scenario, a variety of spectra and spectral trends are expected (see, e.g. Brunetti \& Jones 2014). Compelling evidence is now available that directly links radio relics with cluster shocks observed at X-rays and $\mathrm{mm} / \mathrm{sub}$-mm wavelengths (e.g., Akamatsu et al. 2013; Planck Collaboration et al. 2013; Erler et al. 2015; Eckert et al. 2016; Urdampilleta et al. 2018). According to

\footnotetext{
1 The other two galaxy clusters found to be significant outliers are A1213 (Giovannini et al. 2009) and CLG 0217+70 (Brown et al. 2011). Zhang et al. (2020) recently demonstrate that by adopting a new revised redshift estimate, the galaxy cluster CLG $0217+70$ is not anymore overluminous in radio compared to X-ray, while the case of A1213 is still under investigation.
}

these findings, the emission observed in radio seems to be explained if cosmic-ray electrons are accelerated up to $\sim \mathrm{GeV}$ energies through diffusive shock acceleration (DSA Drury 1983; Blandford \& Ostriker 1978) either from the thermal pool, as proposed first by Ensslin et al. (1998), or from supra-thermal/relativistic plasma (e.g., Markevitch et al. 2005 and Kang et al. 2012).

Precious insights on the origin of these sources come from the spectral analysis of their properties over a wide frequency range and from the comparison of observations with theoretical expectations and simulations. Radio halos are characterized by steep-spectra $\left(S_{v} \propto\right.$ $v^{-\alpha}$, with $\alpha \simeq 1-1.4$, where $S_{v}$ is the flux density at the observing frequency $v$ and $\alpha$ is the spectral index). Detailed spatially-resolved spectral index images have been produced for a number of radio halos, revealing different behaviours. Indications of radial steepening have been found in a few clusters (e.g., Giovannini et al. 1993; Feretti et al. 2004; Pearce et al. 2017), while other radio halos show no clear trend with either uniform (e.g., Vacca et al. 2014; Rajpurohit et al. 2018; Hoang et al. 2019) or complex (e.g., Giacintucci et al. 2005; Orrú et al. 2007; Kale \& Dwarakanath 2010; Shimwell et al. 2014; Rajpurohit et al. 2020; Botteon et al. 2020) distributions. Radio relics are also characterized by steep-spectra ( $\alpha \simeq 1-1.5)$, and detailed spectral index images show a steepening transversely to their elongation towards the cluster center (e.g., Clarke \& Ensslin 2006; de Gasperin et al. 2015; Di Gennaro et al. 2018). The steepening is interpreted as synchrotron and inverse Compton losses in the shock downstream region.

In this paper we present new $144 \mathrm{MHz}$ LOw Frequency ARray (LOFAR) observations and new 1-2 GHz Jansky Very Large Array (VLA) observations of the galaxy cluster A523 which hosts a powerful diffuse source associated with the ICM, historically classified as a radio halo. Its radio power at $1.4 \mathrm{GHz}$ makes it one of the most significant outliers in the scaling relations between observables derived from multi-wavelength observations of galaxy clusters: it has a morphology that is different and offset from the thermal gas, and it has polarized emission at $1.4 \mathrm{GHz}$ typically difficult to observe for this class of sources. We study the spectral behaviour of the emission between $144 \mathrm{MHz}$ and $2 \mathrm{GHz}$ to better understand the formation mechanism of this source and compare the radio emission with observations at X-ray wavelengths. In $\$ 2$ we summarize the present knowledge on this cluster, in $\S 3$ we describe the data used for the analysis. In $\S 4$ we present the new images of the cluster, in $\$ 5$ the spectral behaviour and in $\$ 6$ a comparison between the radio and $\mathrm{X}$-ray derived cluster properties. Finally, in $\$ 7$ and in $\$ 8$ we discuss the nature of the system and draw our conclusions. Throughout, we use a $\Lambda$ CDM cosmology with $H_{0}=67.4 \mathrm{~km} / \mathrm{s} / \mathrm{Mpc}, \Omega_{0}=0.315$ and $\Omega_{\Lambda}=0.685$ (Planck Collaboration et al. 2020). With this cosmology, at the distance of A523 ( $z=0.104$, luminosity distance $499 \mathrm{Mpc})$, $1^{\prime \prime}$ corresponds to $1.98 \mathrm{kpc}$.

\section{THE GALAXY CLUSTER A523}

A523 is a nearby cluster $(z=0.1040 \pm 0.0004$, Girardi et al. 2016) with an estimated $M_{\mathrm{X}, 500} \approx 2.2-3.6 \times 10^{14} M_{\odot}$ Cova et al. 2019 , where $M_{\mathrm{X}, 500}$ is the mass derived from X-observables within $R_{500}{ }^{2}$. A523 is a disturbed system with an ongoing merger along the SSWNNE direction between two subclusters (Girardi et al. 2016; Golovich

\footnotetext{
2 We refer to $R_{\Delta}$ as the radius of a sphere within which the mean mass density is $\Delta$ times the critical density at the redshift of the galaxy cluster system. $M_{\Delta}$ is the mass contained in $R_{\Delta}$.
} 
Table 1. Radio observations used in this work.

\begin{tabular}{ccccccccc}
\hline \hline $\begin{array}{c}\text { RA } \\
\text { h:m:s } \\
(J 2000)\end{array}$ & $\begin{array}{c}\text { Dec } \\
0:^{\prime \prime} \\
(\mathrm{J} 2000)\end{array}$ & Instrument & $\begin{array}{c}v \\
(\mathrm{MHz})\end{array}$ & $\begin{array}{c}\text { Bandwidth } \\
(\mathrm{MHz})\end{array}$ & Config. & Date & $\begin{array}{c}\text { Duration } \\
(\mathrm{h})\end{array}$ & Project \\
\hline \hline 04:57:06.3 & $+08: 56: 53.7$ & LOFAR & 144 & 48 & HBA DUAL & $31-10-2018$ & 4 & LC10_024 \\
$04: 57: 06.3$ & $+08: 56: 53.7$ & LOFAR & 144 & 48 & HBA DUAL & $11-10-2018$ & 4 & LC10_024 \\
\hline $04: 59: 10.0$ & $+08: 49: 00.0$ & VLA & 1.5 & 1000 & $\mathrm{C}$ & $06-07-2013$ & 1.1 & $13 \mathrm{~A}-168$ \\
$04: 59: 10.0$ & $+08: 49: 00.0$ & VLA & 1.5 & 1000 & $\mathrm{D}$ & $29-01-2013$ & 1.1 & $13 \mathrm{~A}-168$ \\
\hline \hline
\end{tabular}

et al. 2019). A secondary merger is likely present along the ESEWNW direction (Cova et al. 2019). The cluster is known to host a powerful extended synchrotron source, classified as a radio halo because it permeates both the merging clumps and does not show transverse flux asymmetry typical of radio relics (Giovannini et al. 2011). Girardi et al. (2016) derived a flux density of the source at $1.4 \mathrm{GHz}$ of $72 \pm 3 \mathrm{mJy}$. According to Einasto et al. (2001), A523 belongs to the supercluster SCL 62 that includes the clusters A525, A515, A529, A532, all of which lie at a similar redshift. In this context, the supercluster has been recently targeted to search for diffuse emission in and beyond galaxy clusters with the Sardinia Radio Telescope (SRT) by Vacca et al. (2018b) who found patches of diffuse emission of unknown origin. A fraction of these turned out to be Galactic $\mathrm{H}-\alpha$ regions and blending of discrete sources (Hodgson et al. 2020). Follow up studies are still in progress for the remaining regions.

A detailed multi-wavelength study including optical, X-ray, and radio data at $1.4 \mathrm{GHz}$ has enabled an accurate characterization of the cluster dynamical and non-thermal properties, revealing that the radio halo emission is mainly elongated perpendicular to the merging axis defined by the optical and X-ray observations, with a clear offset between the peaks of the radio and the X-ray brightness distribution of about $310 \mathrm{kpc}$ (Girardi et al. 2016). Unlike other radio halos, the source in A523 does not show a strong point-to-point correlation between the $1.4 \mathrm{GHz}$ radio and $\mathrm{X}$-ray brightness distribution but it is rather characterized by a broad intrinsic scatter between these two quantities (Cova et al. 2019). Overall, its radio power ${ }^{3}$ at $1.4 \mathrm{GHz}$ is $P_{1.4 \mathrm{GHz}}=2.2 \times 10^{24} \mathrm{~W} / \mathrm{Hz}$, while the X-ray luminosity is $L_{\mathrm{X}, 500}[0.1-2.4]=1.5 \times 10^{44} \mathrm{erg} / \mathrm{s}$ (Girardi et al. 2016; Cova et al. 2019). The $1.4 \mathrm{GHz}$ radio emission appears to be higher by a factor $\sim 6-18$ than expected from the X-ray luminosity of the system according to the best-fit scaling relations presented in Yuan et al. (2015). We use for the redshift of this cluster $z=0.1040 \pm 0.0004$ (Girardi et al. 2016), derived through a detailed optical study based on 132 spectroscopic redshifts of which 80 related to cluster members and later confirmed by Golovich et al. (2019). This makes it very unlikely that an incorrect estimate of the redshift could be the reason of the higher radio power.

It might be that the cluster emission is overly bright in the radio because of a third subcluster merging in the direction perpendicular to the main merger and causing additional turbulence, as proposed by Cova et al. (2019) on the basis of new XMM-Newton and Nustar observations. Other clusters undergoing multiple mergers have been found to host radio halos, but these radio halos are not over-luminous in radio with respect to X-rays (e.g., Bonafede et al. 2009). The peculiarity of A523 is apparent also in its location on the $P_{1.4 \mathrm{GHz}}$ - $M_{\mathrm{X}, 500}$ correlation. By considering the $P_{1.4 \mathrm{GHz}}-M_{\mathrm{X}, 500}$ correla-

\footnotetext{
${ }^{3}$ k-correction was applied with a spectral index $\alpha=1.2$.
}

tion for radio halos found by Yuan et al. (2015), we derive a mass $M_{\mathrm{X}, 500} \approx 6-10 \times 10^{14} M_{\odot}$, larger than the estimated mass by Cova et al. (2019) even when the uncertainties in the radio power and in the correlation have been taken into account.

A strongly polarized signal $(\sim 15-20 \%)$, associated with the radio halo, has been detected by Girardi et al. (2016) across most of the radio halo extension. Numerical three-dimensional simulations demonstrate that an intracluster magnetic field with a strength $\left\langle B_{0}\right\rangle \simeq 0.5 \mu \mathrm{G}$ at the center of the cluster and fluctuating over a range of scales between a few kpc up to $1 \mathrm{Mpc}$ is consistent with both the total intensity and polarized emission of the radio halo (Girardi et al. 2016). The magnetic field has been constrained by the fractional polarization of the diffuse emission, and is therefore relatively independent of the relativistic electron population. Such a magnetic field auto-correlation scale is much larger than the resolution of radio observations $(\sim 130 \mathrm{kpc})$, preventing the beam depolarization of the radio signal that usually hinders the detection of radio halo polarized emission (Govoni et al. 2013). The value of the magnetic field strength derived by Girardi et al. (2016) is consistent with the results from upper limits on inverse Compton emission of the cluster by Cova et al. (2019), who derive a magnetic field larger than $0.2 \mu \mathrm{G}$ over the whole radio halo region and larger than $0.8 \mu \mathrm{G}$ when only the brightest region is considered.

\section{RADIO OBSERVATIONS AND DATA REDUCTION}

For our analysis, we use new LOFAR data at $120-168 \mathrm{MHz}$ and new VLA observations in the frequency range $1-2 \mathrm{GHz}$, as described in the following subsections.

\subsection{LOFAR observations}

We present LOFAR data (SAS ID 682162,672474) obtained through the observing program LC10_024 (PI V. Vacca). A summary of the observations is reported in Table 1 . The observations have been carried out with the instrument in interferometer mode in the HBA Dual Inner configuration, with the full (core, remote and international stations) LOFAR array. The study presented in this paper is based on the data from the core and remote stations only. We used a $48 \mathrm{MHz}$ (120-168 MHz) bandwidth with a total of 231 sub-bands per beam. The observations exploit the multi-beam capability of the HBA and were conducted together with the LoTSS observations through the co-observing program offered by the observatory. The observations presented here correspond to the LoTSS pointing P074+09. Due to the low declination of the source $\left(\sim 8.8^{\circ}\right)$, the observation has been split in two $4 \mathrm{~h}$-blocks to ensure the elevation of the target is higher than $30^{\circ}$ throughout the observation.

To solve for the complex gains, and to calibrate the bandpass and amplitude, a direction-independent calibration step has been 
Table 2. Details of the radio brightness images presented in this work. Col. 1, 2: central frequency and bandwidth; Col. 3: uv-range; Col. 4: resolution; Col. 5: maximum angular scale accessible with the observations; Col. 6: sensitivity; Col. 7: robust; Col. 8: figure/table where the image has been used.

\begin{tabular}{cccccccc}
\hline \hline $\begin{array}{c}\text { Frequency } \\
\mathrm{MHz}\end{array}$ & $\begin{array}{c}\text { Bandwidth } \\
\mathrm{MHz}\end{array}$ & $\begin{array}{c}\text { uv-range } \\
\lambda\end{array}$ & $\begin{array}{c}\text { Beam } \\
\prime \times \prime \prime\end{array}$ & $\begin{array}{c}\text { Max scale } \\
\circ\end{array}$ & $\begin{array}{c}\sigma \\
\text { mJy/beam }\end{array}$ & Robust & Figure/Table \\
\hline 144 & 48 & all & $9 \times 9$ & 1.2 & 0.35 & -0.5 & Fig. 1 \\
144 & 48 & $980-16980$ & $13.5 \times 13.5$ & 0.06 & 0.5 & -1 & Table3 \\
144 & 48 & all & $20 \times 20$ & 1.2 & 0.4 & -0.5 & Fig. 2 \\
144 & 48 & $158-16980$ & $20 \times 20$ & 0.36 & 0.45 & -0.5 & Fig. 5 \\
144 & 48 & all & $65 \times 65$ & 1.2 & 1.3 & -0.5 & Fig. 3 \\
144 & 48 & $158-4835$ & $65 \times 65$ & 0.36 & 0.9 & -0.5 & Fig. 5 \\
144 & 48 & $196-4835$ & $65 \times 65$ & 0.29 & 0.9 & -0.5 & Fig. 4 \\
1410 & 192 & $980-16980$ & $13.5 \times 13.5$ & 0.06 & 0.09 & -1 & Table3 \\
1410 & 192 & $158-16980$ & $20 \times 20$ & 0.36 & 0.075 & -0.5 & Fig. 5 \\
1410 & 192 & all & $65 \times 65$ & 0.36 & 0.14 & 0.5 & Fig. 3 \\
1410 & 192 & $158-4835$ & $65 \times 65$ & 0.36 & 0.13 & -0.5 & Fig. 5 \\
1410 & 192 & $196-4835$ & $65 \times 65$ & 0.29 & 0.12 & -0.5 & Fig. 4 \\
1782 & 320 & $980-16980$ & $13.5 \times 13.5$ & 0.06 & 0.08 & -1 & Table 3 \\
1782 & 320 & all & $65 \times 65$ & 0.29 & 0.11 & 0.5 & Fig. 3 \\
1782 & 320 & $196-4835$ & $65 \times 65$ & 0.29 & 0.11 & -0.5 & Fig. 4 \\
\hline \hline
\end{tabular}

done. The non-directional calibration includes radio frequency interference (RFI) and bright off-axis source removal, as well as clock-total electron content separation based on the calibrator data (see e.g., van Weeren et al. 2016a). During this step the calibrators J0813+4813 (3C196) and J0542+4951 (3C147) have been used as reference respectively for each of the two observing blocks. Direction-independent calibration of the data has been done with pre-factor that takes $1 \mathrm{~s}$ and $16 \mathrm{ch} / \mathrm{sb}$ and averages down to $8 \mathrm{~s}$ and $2 \mathrm{ch} / \mathrm{sb}$ (van Weeren et al. 2016a; de Gasperin et al. 2019). These direction-independent calibrated datasets have then been processed with the direction-dependent ddf-pipeline used by the LOFAR Surveys Key Science Project (KSP) for reduction of the LoTSS survey (Tasse et al. 2021) ${ }^{4}$. The ddf-pipeline is based on DDFacet (Tasse et al. 2018) and KillMS (Tasse 2014a,b; Smirnov \& Tasse 2015) and repeats several iterations of direction-dependent self-calibration towards 45 directions of the sky (i.e. facets), with an inner cut on the recorded visibilities of $0.1 \mathrm{~km}$, corresponding to $\sim 48 \lambda$ at the central frequency of the observations. Shorter baselines have been excluded because of RFI. We performed a further refining of the data in the region where the cluster is located through amplitude and phase selfcalibration. Flux contributions from sources outside a square box with sides of length $0.4^{\circ}$ centered at approximately the location of the X-ray peak of the cluster (RA 04h:59m:07s and Dec $08^{\circ}: 45^{\prime}: 00^{\prime \prime}$ ) have been subtracted in the $u v$ plane using the direction-dependent calibrations and final ddf-pipeline model, a phase-shift has been applied to locate the target in the phase center, and finally a correction for the LOFAR station beam towards this direction has been applied (this procedure is detailed in van Weeren et al. 2021). We reimage the data that are calibrated in the direction of our target using wsclean2.10 (Offringa et al. 2014). A post-processing step, summarised in Hardcastle et al. (2021) and which will be described in more detail in Shimwell et al. in prep., was used to scale the wide-field image of our pointing to align the fluxes with the same technique used for LoTSS-DR2 which is itself aligned with the 6C survey (Hales et al. 1988, 1990) through a comparison with the NRAO VLA Sky Survey (NVSS, Condon et al. 1998). The flux scale of the extracted data which, as described above, underwent further processing, was then aligned with the wide field by applying an overall multiplicative factor of 1.720 to the final images made from the extracted data to ensure alignment consistent with the flux scaling strategy used for the LoTSS Data Release 2.

In order to investigate the emission from discrete sources as well as from the radio halo, we produced images at low- and high-spatial resolution. At this declination the beam is not circular and, in order to better compare our results with $1.4 \mathrm{GHz}$ observations we convolved the high resolution image with a circular beam of $9^{\prime \prime}$ and $13.5^{\prime \prime}$, while circular beams of $20^{\prime \prime}$ and $65^{\prime \prime}$ have been used to restore the images at lower resolution. We note that these images have been produced including all baselines starting from $48 \lambda$. Shorter baselines have been excluded because of RFI, as noted above. Details about the images presented in the following are given in Table 2. In the following, we assume an uncertainty on the LOFAR flux density scale of $20 \%$, as done for LoTSS (Shimwell et al. 2019).

\subsection{VLA observations}

We present new VLA data in the frequency range 1-2 GHz in C and D configuration obtained with a mosaic of six pointings in the context of the observing program 13A-168 (PI M. Murgia). The details of the observations are reported in Table $1^{5}$. The data were reduced following standard procedures using the NRAO's Astronomical Image Processing System (AIPS) package. The data were collected in spectral line mode in full Stokes, with a total initial bandwidth of $1 \mathrm{GHz}$ subdivided in 16 spectral windows. Each spectral window is $64 \mathrm{MHz}$ wide with 64 channels, and Hanning smoothing was applied before bandpass calibration. The source J0319+4130 (3C84) was used as a bandpass and polarization leakage calibrator, the source J0137+3309 (3C48) was used as a flux density calibrator, and the nearby source J0459+0229 was observed for complex gain calibration. The flux density scale by Perley \& Butler (2017) was adopted. RFI flagging has been applied by excision of data with values clearly exceeding the source flux ( $\gtrsim 600 \mathrm{mJy}$ ). Following calibration, the data were spectrally averaged to 16 channels of $4 \mathrm{MHz}$ per $64 \mathrm{MHz}$ wide spectral window. Spectral windows from 0 to 5,9 and 10 were too severely 

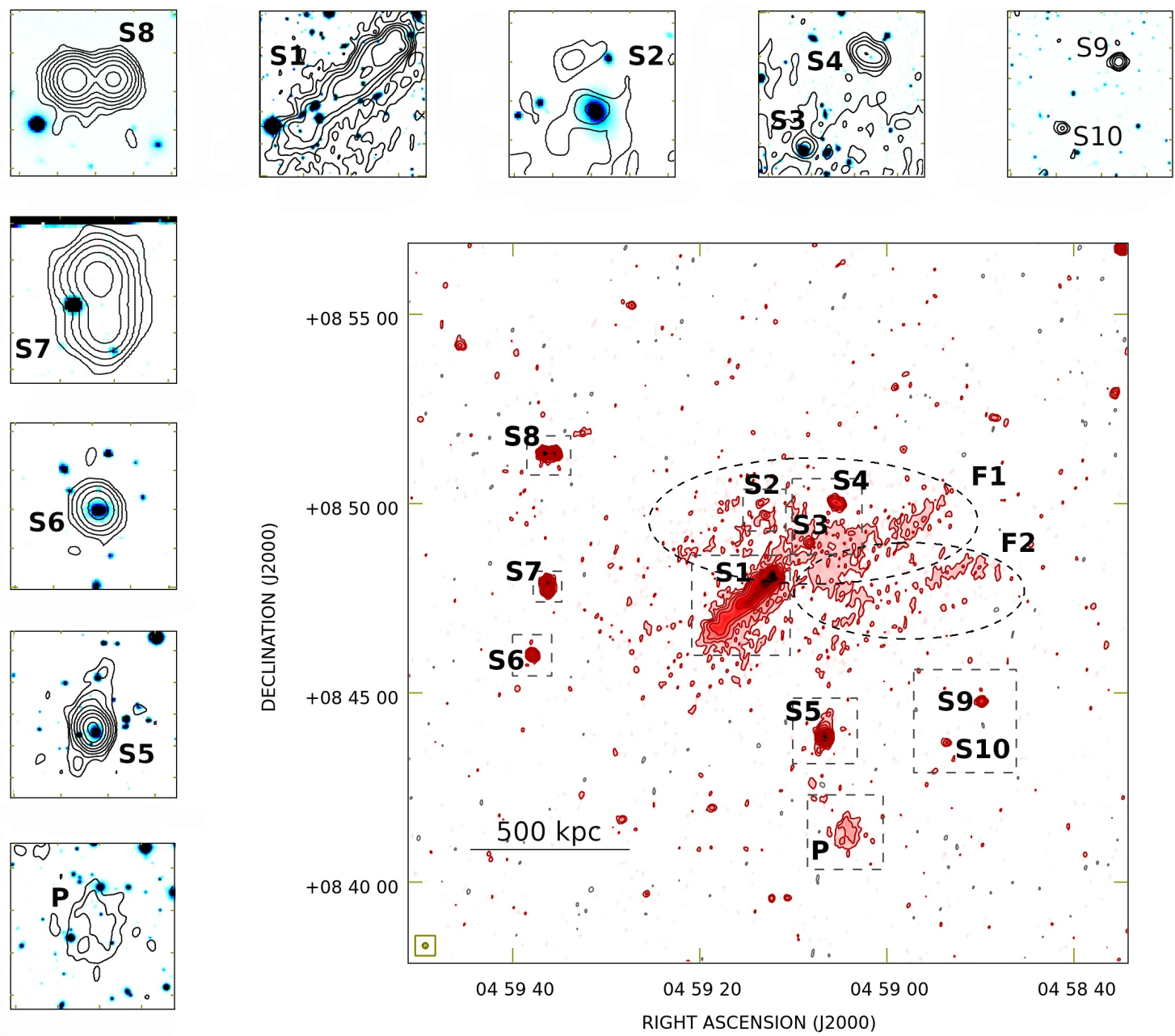

Figure 1. Main panel: LOFAR high-resolution image $\left(9^{\prime \prime} \times 9^{\prime \prime}\right)$ of the central region of the galaxy cluster A523 in contours and colors. Red contours start at $3 \sigma$ $(\sigma=0.35 \mathrm{mJy} / \mathrm{beam})$ and scale by a factor 2, gray contours are drawn at $-3 \sigma$. The horizontal black bar corresponds to $500 \mathrm{kpc}$ at the chosen cosmology and the synthesised beam is shown in the bottom left. The dashed ellipses identify the position of the two filaments F1 and F2. Left and top panels: Zoom on the discrete sources in the field where the radio contours at 9" are superimposed on the optical image from the INT r-band by Girardi et al. (2016), see the text for more details. Positive radio contours are the same as in the large panel. The position of the small panels is marked in the main panel by dashed gray boxes.

affected by RFI to provide useful information, so we disregard them in the following.

Surface brightness images were produced using the Common Astronomy Software Applications (CASA) package (task tclean). The final images have been produced using spectral windows from 6 to 8 and from 11 to 15 , including the full $u v$-range available, unless differently stated. Circular restoring beams of $13.5^{\prime \prime}, 20^{\prime \prime}$ and $65^{\prime \prime}$ were used to restore the images in $\mathrm{C}, \mathrm{C}+\mathrm{D}$, and in $\mathrm{D}$ configurations, respectively. Details about the images presented in the following are given in Table 2. In the following, we assume an uncertainty on the VLA flux density scale of $2.5 \%$, in agreement with Perley \& Butler (2013).

Everywhere in the text, unless differently stated, the overall uncertainties on the radio brightness and flux density include both the statistical uncertainty and the appropriate uncertainty in the flux density scale.

\section{CONTINUUM IMAGES}

In Fig. 1 we show the $144 \mathrm{MHz}$ cluster emission with $9^{\prime \prime}$ resolution. A number of radio galaxies, labelled S1-S10, are present in the field embedded in the diffuse emission and with peaks in the radio brightness higher than $8 \sigma$. Zoom in's on these radio galaxies are shown in the left and top panels, where the radio contours are superimposed on the optical image from the Isaac Newton Telescope (INT) r-band by Girardi et al. (2016). These authors identify the head tail S1 with the member-galaxy ID 68, S3 with a point-like source with unknown redshift, S4 with a likely background galaxy, and S5 with the second $\mathrm{BCG}$ of the cluster. Using the optical information from the same authors, S2 appears to be the radio counterpart of the brightest cluster galaxy, previously undetected in radio, and S6 the dominant galaxy in the galaxy population of the ESE region, likely in the background at $\mathrm{z} \sim 0.14$.

Two filaments of size $\sim 6^{\prime} \times 1^{\prime}$ (corresponding to about $710 \mathrm{kpc} \times 120 \mathrm{kpc}$ ) and with higher surface brightness than their surrounding regions of radio emission can be identified and are labelled respectively F1 and F2 (see dashed ellipses in Fig. 1). F1 occupies the region to the north-west of $\mathrm{S} 1$, with a few embedded radio sources 


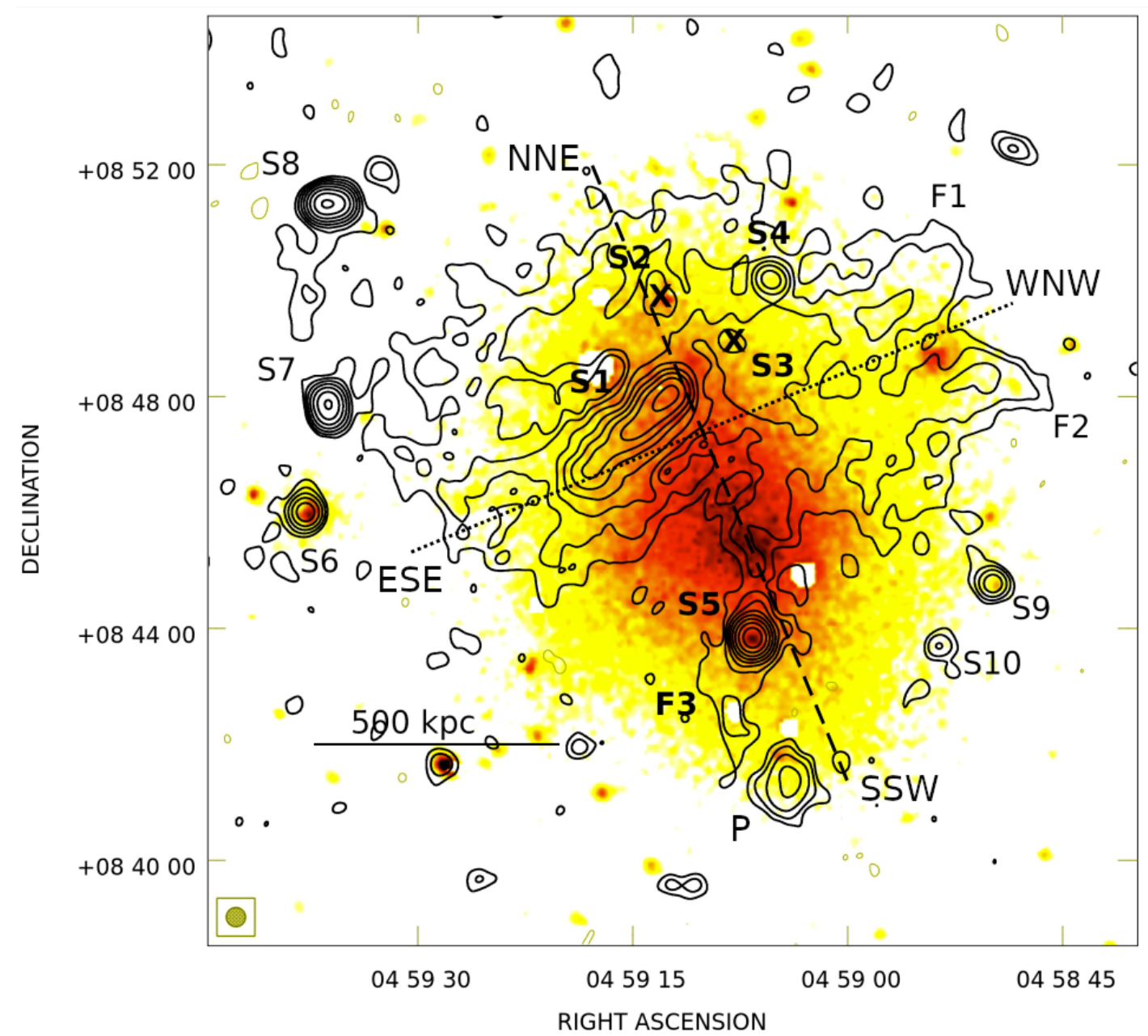

Figure 2. Radio emission from the galaxy cluster A523 at $144 \mathrm{MHz}$ with LOFAR at $20^{\prime \prime}$. Black contours start at $3 \sigma(\sigma=0.4 \mathrm{mJy} / \mathrm{beam})$ and scale by a factor 2 , while negative $3 \sigma$ contours are drawn in army green. The beam is shown in the bottom left of the image and the horizontal black bar corresponds to $500 \mathrm{kpc}$ at the chosen cosmology. The radio emission is overlaid on the X-ray image of the cluster from XMM-Newton (Cova et al. 2019), see the text for more details. The dashed and the dotted lines show respectively the axis of the primary and of the secondary merger.

(S2, S3, S4). F2 is located west of the central AGN S1. No other similar structures are seen elsewhere in the image, suggesting that these filaments are unlikely to be artefacts related to calibration or imaging but rather are real structures. Further south, a new roundish patch of emission, labelled $\mathrm{P}$, is detected at RA $04 \mathrm{~h} 59 \mathrm{~m} 04 \mathrm{~s}$ and Dec $08^{\circ} 41^{\prime} 21^{\prime \prime}$, with an average brightness that is higher than the brightest regions of the filaments.

In Fig. 2 we show the radio image of the cluster at $144 \mathrm{MHz}$ obtained with LOFAR at $20^{\prime \prime}$. The radio image is superimposed on the background-subtracted and exposure-corrected XMM-Newton image, with point sources removed. The X-ray image has been obtained with the combination of data from the three EPIC instruments in the soft $0.5-2.5 \mathrm{keV}$ band for a total exposure time of about $220 \mathrm{ks}$ and was recently published by Cova et al. (2019). Dashed and dotted lines indicate respectively the primary (SSW-NNE) and secondary (ESE-WNW) merger axis. The diffuse radio source extends further than filaments F1 and F2, and a third filament, F3, is visible, with a size of $7^{\prime} \times 1^{\prime}$ (i.e., $830 \mathrm{kpc} \times 120 \mathrm{kpc}$ ), that embraces S5 and terminates near the roundish patch of emission P. Overall, the diffuse emission sits in the northern part of the thermal gas distribution, is characterized by an E-W linear morphology that is approximately perpendicular to the axis of the main merger in the SSW-NNE direction, cuts through the central part of the X-ray region, and encompasses the discrete sources S1, S2, S3, S4, and S5 (see Fig. 2).

\section{Flux density of point sources}

In Table 3, we report the position and flux densities of the discrete sources S1-S10 at $144 \mathrm{MHz}, 1.410 \mathrm{GHz}$, and $1.782 \mathrm{GHz}$ estimated from the images at $13.5^{\prime \prime}$, obtained by applying the same $u v$-range and weighting-scheme (robust=-1) at the three frequencies. As in Vacca et al. (2018b), we model the sources with a 2-dimensional elliptical Gaussian sitting on a plane. Overall, there are nine free parameters of the fit: the $\mathrm{x}, \mathrm{y}$ coordinates in the sky of the centre of the Gaussian, the full width half maximum along the two axes, the position angle, the amplitude, and the three components of the direction normal to the plane. The non-zero baseline fit ensures that any contribution from the diffuse emission, if present, is not absorbed into the flux density of the sources. The uncertainty has been estimated by adding in quadrature the uncertainty derived from the fit procedure and the flux scale uncertainty at the corresponding frequency $(20 \%$ at $144 \mathrm{MHz}$ and of $2.5 \%$ at 1.410 and $1.782 \mathrm{GHz}$ ). This procedure was used for all the discrete sources except the central AGN S1. Being an extended source, we determined the flux density by applying a $3 \sigma$ 
Table 3. Spectral index of point sources embedded in the diffuse emission. Col. 1: Source name (see Fig. 1); Col. 2,3: RA and Dec corresponding to the peak of the source at $144 \mathrm{MHz}$; Col. 4, 5, 6: Flux density at $144 \mathrm{MHz}, 1.410 \mathrm{GHz}$, and $1.782 \mathrm{GHz}$ respectively (this work); Col. 7: Spectral index derived from the flux density values at $144 \mathrm{MHz}$ and $1.410 \mathrm{GHz}$ (this work); Col. 8, 9, 10: Flux density from TGSS (147 MHz) and NVSS (1.4 GHz) and the corresponding spectral index as given by de Gasperin et al. (2018).

\begin{tabular}{|c|c|c|c|c|c|c|c|c|c|}
\hline Source & $\begin{array}{l}\text { RA (J2000) } \\
\text { hh:mm:ss }\end{array}$ & $\begin{array}{c}\text { Dec (J2000) } \\
\circ::^{\prime \prime}\end{array}$ & $\begin{array}{c}S_{144 \mathrm{MHz}} \\
\mathrm{mJy}\end{array}$ & $\begin{array}{c}S_{1.410 \mathrm{GHz}} \\
\mathrm{mJy}\end{array}$ & $\begin{array}{c}S_{1.782 \mathrm{GHz}} \\
\mathrm{mJy}\end{array}$ & $\alpha$ & $\begin{array}{c}S_{\mathrm{TGSS}} \\
\mathrm{mJy}\end{array}$ & $\begin{array}{c}S_{\mathrm{NVSS}} \\
\mathrm{mJy}\end{array}$ & $\alpha_{\mathrm{dG} 18}$ \\
\hline S1 & $04: 59: 12.3$ & $+08: 48: 04$ & $971.98^{1} \pm 194.43$ & $104.19^{1} \pm 2.68$ & $89.25 \pm 2.30$ & $0.98 \pm 0.09$ & $688.03 \pm 13.90$ & $121.71 \pm 1.22$ & $0.77 \pm 0.01$ \\
\hline S2 & 04:59:13.0 & $+08: 49: 41$ & $5.81 \pm 1.17$ & $0.54 \pm 0.03$ & $0.23 \pm 0.02$ & $1.04 \pm 0.09$ & - & - & - \\
\hline S3 & 04:59:08.3 & $+08: 48: 58$ & $10.88 \pm 2.18$ & $1.04 \pm 0.04$ & $0.93 \pm 0.03$ & $1.03 \pm 0.09$ & - & - & - \\
\hline S4 & 04:59:05.4 & $+08: 50: 01$ & $32.48 \pm 6.50$ & $4.44 \pm 0.11$ & $4.55 \pm 0.12$ & $0.87 \pm 0.09$ & - & - & - \\
\hline S5 & 04:59:06.6 & $+08: 43: 50$ & $313.68 \pm 62.74$ & $61.74 \pm 1.54$ & $55.84 \pm 1.40$ & $0.71 \pm 0.09$ & $213.91 \pm 11.10$ & $61.33 \pm 1.01$ & $0.55 \pm 0.02$ \\
\hline S6 & 04:59:37.9 & $+08: 46: 01$ & $34.65 \pm 6.93$ & $6.91 \pm 0.17$ & $5.78 \pm 0.15$ & $0.71 \pm 0.09$ & $0.0 \pm 0.0$ & $6.11 \pm 1.02$ & $0.52 \pm 0.00$ \\
\hline S7 & $04: 59: 36.3$ & $+08: 47: 54$ & $112.34 \pm 22.47$ & $10.87 \pm 0.27$ & $8.20 \pm 0.21$ & $1.02 \pm 0.09$ & $77.54 \pm 11.05$ & $8.69 \pm 1.02$ & $0.97 \pm 0.09$ \\
\hline S8 & $04: 59: 36.5$ & $+08: 51: 20$ & $238.71 \pm 47.74$ & $22.14 \pm 0.57$ & $17.35 \pm 0.44$ & $1.04 \pm 0.09$ & $144.93 \pm 11.41$ & $19.54 \pm 1.00$ & $0.89 \pm 0.04$ \\
\hline S9 & 04:58:49.9 & $+08: 44: 46$ & $16.27 \pm 3.26$ & $3.21 \pm 0.09$ & $2.83 \pm 0.07$ & $0.71 \pm 0.09$ & $0.0 \pm 0.0$ & $5.30 \pm 1.00$ & $0.68 \pm 0.00$ \\
\hline $\mathrm{S} 10$ & 04:58:53.6 & $+08: 43: 41$ & $4.87 \pm 0.98$ & $0.44 \pm 0.02$ & $0.48 \pm 0.02$ & $1.05 \pm 0.09$ & - & - & - \\
\hline
\end{tabular}

${ }^{1}$ These flux density values include the contribution of the diffuse emission that amounts to about $35 \mathrm{mJy}$ at $144 \mathrm{MHz}$ and to about $2 \mathrm{mJy}$ at $1.4 \mathrm{GHz}$.

sensitivity threshold to the LOFAR image $(\sigma=0.5 \mathrm{mJy} / \mathrm{beam})$ and blanked the two VLA images in the same regions as the LOFAR one. We refer to $\S 5.3$ for spectral studies of these sources.

\section{Flux density of diffuse emission}

In panel (a) of Fig. 3 we show in black contours the LOFAR image of the cluster at $144 \mathrm{MHz}$ overlaid on the same X-ray image as in Fig. 2, while in panel (b) the LOFAR image with the regions used to mask discrete sources. In the panels (c) and (d), we show on the left the new VLA image at $1.410 \mathrm{GHz}$ in contours overlaid on the LOFAR image in gray scale and on the right the new VLA image at $1.782 \mathrm{GHz}$. All the radio images are at $65^{\prime \prime}$ resolution. Comparison of the LOFAR and VLA images shows that the radio halo appears more extended at lower frequencies. The LOFAR image at $65^{\prime \prime}$ indeed reveals a size of the source of about $15^{\prime}$, corresponding to $1.8 \mathrm{Mpc}$ at the cluster distance. The maximum angular scale accessible with our data is about $1.2^{\circ}, 22^{\prime}$, and $17^{\prime}$ respectively at $144 \mathrm{MHz}, 1.410 \mathrm{GHz}$ and $1.782 \mathrm{GHz}$, as reported in Table 2. The sensitivities $\sigma$ at these three frequencies and spatial resolution are $1.3 \mathrm{mJy} / \mathrm{beam}, 0.14 \mathrm{mJy} / \mathrm{beam}$, and $0.11 \mathrm{mJy} / \mathrm{beam}$. A brightness of $3.9 \mathrm{mJy} / \mathrm{beam}$ at $144 \mathrm{MHz}$ (i.e., at $3 \sigma$ ), as the faintest regions of the emission in the LOFAR image, corresponds to $0.400-0.160 \mathrm{mJy} / \mathrm{beam}$ at $1.410 \mathrm{GHz}$ and to 0.315 $0.115 \mathrm{mJy} /$ beam at $1.782 \mathrm{GHz}$, for typical spectral index values $(\alpha=1-1.4)$. A sensitivity $\sigma$ of $0.133-0.053 \mathrm{mJy} / \mathrm{beam}$ at $1.410 \mathrm{GHz}$ and of $0.105-0.038 \mathrm{mJy} / \mathrm{beam}$ at $1.782 \mathrm{GHz}$ is needed to detect this emission, while the sensitivity in our images is $0.14 \mathrm{mJy} / \mathrm{beam}$ and $0.11 \mathrm{mJy} /$ beam respectively at $1.410 \mathrm{GHz}$ and $1.782 \mathrm{GHz}$, see Table 2. The larger extent detected at LOFAR frequencies compared to VLA higher frequency images is therefore likely due to the better sensitivity to steep spectrum emission of LOFAR.

A faint region of diffuse emission in the north-east and in the south of the cluster emerges in the $144 \mathrm{MHz}$ image at $65^{\prime \prime}$, along the main merger axis (see arrows in Fig. 3), panel (c), along the optical and X-ray distribution of the system, see Fig. 1 in Girardi et al. (2016). Consequently, at this frequency the emission along the SSW-NNE axis is more prominent than at $\sim 1-2 \mathrm{GHz}$. At $1.410 \mathrm{GHz}$ only the brightest regions along the ESE-WNW direction survive, while the emission in the south, in the south-east and in the north-east falls below the noise. At $1.782 \mathrm{GHz}$, the diffuse emission dims further, the filaments F1, F2, part of F3 and a bridge of radio emission connecting S1 to S6 only are still apparent. In order to measure the flux densities at the three frequencies, we masked embedded compact sources as shown in Fig. 3 (panel b). We assume that the halo extends over the location of embedded compact sources. In these masked regions we assume the halo radio brightness is equal to its mean radio brightness over the entire unmasked diffuse source. Overall, we derive a flux density at $144 \mathrm{MHz}$ of $S_{144 \mathrm{MHz}}=(1.52 \pm 0.31) \mathrm{Jy}$, including the southern patch. The uncertainty takes into account the uncertainty on the flux scale and the image noise. By using the same method to extrapolate over embedded masked regions, at $1.410 \mathrm{GHz}$ we measure a flux density of the diffuse emission of $S_{1.410 \mathrm{GHz}}=(70 \pm 2) \mathrm{mJy}$, in agreement within $\approx 0.8 \sigma$ with the flux density derived by Girardi et al. (2016) $S_{1.4 \mathrm{GHz}}=(72 \pm$ 3) $\mathrm{mJy}$, while at $1.782 \mathrm{GHz}$ we measure a flux density $S_{1.782 \mathrm{GHz}}=$ $(42 \pm 1)$ mJy. These flux densities have been derived considering all the available $u v$-range and applying a sensitivity cut of $3 \sigma$ at the corresponding frequency, so they refer to the full size of the source at those frequencies.

In panel (a) of Fig. 3, the patch $\mathrm{P}$ is more extended compared to Fig. 2 and blended with S5 and with the rest of the diffuse emission. In order to better discriminate the emission associated with this source, we used the $20^{\prime \prime}$ image. We measure a flux density $S_{144 \mathrm{MHz}}=$ $(49 \pm 10) \mathrm{mJy}$ and an average brightness $4.5 \mathrm{mJy} / \mathrm{beam}$. While this patch is clearly detected at $144 \mathrm{MHz}$, only an excess is present at the $3 \sigma$ level $(\approx 0.4 \mathrm{mJy} /$ beam $)$ at $1.410 \mathrm{GHz}$, suggesting a spectral index $\alpha \gtrsim 1.5$. The origin of this emission will be discussed in $§ 5.1$.

\section{SPECTRAL INDEX}

In Fig. 4 we plot the flux density of the diffuse emission at $144 \mathrm{MHz}$, $1.410 \mathrm{GHz}$, and $1.782 \mathrm{GHz}$ versus frequency, derived from images obtained by selecting the same $u v$-range (196-4835 $\lambda$ ), weightingscheme (robust=-0.5), and area on the sky. The $u v_{\text {min }}=196 \lambda$ is imposed by the minimum uv-sampling at $1.782 \mathrm{GHz}$. The corresponding images at $144 \mathrm{MHz}, 1.410 \mathrm{GHz}$, and $1.782 \mathrm{GHz}$ are not shown here. Overall, the spectrum cannot be described with a single power-law, since it steepens towards high frequency. Indeed, as discussed in $\S 4$, at $1.782 \mathrm{GHz}$ only the filaments F1, F2, part of F3, and a bridge connecting S1 to S6 survive, the rest of the emission is buried by the noise. We fit the spectrum with the software synage (Murgia 1996) assuming a power-law. Between $144 \mathrm{MHz}$ and $1.410 \mathrm{GHz}$ we obtain $\alpha_{144 \mathrm{MHz}-1.410 \mathrm{GHz}}=1.2_{-0.2}^{+0.1}$ (see dashed line in Fig. 4), while between $1.410 \mathrm{GHz}$ and $1.782 \mathrm{GHz}$ we obtain $\alpha_{1.410 \mathrm{GHz}-1.782 \mathrm{GHz}}=2.1_{-0.3}^{+0.2}$ (see dotted line in Fig. 4). A similar behaviour has been observed for other radio halos (e.g., Deiss et al. 1997; Thierbach et al. 2003; Xie et al. 2020; Rajpurohit et al. 2021b). 

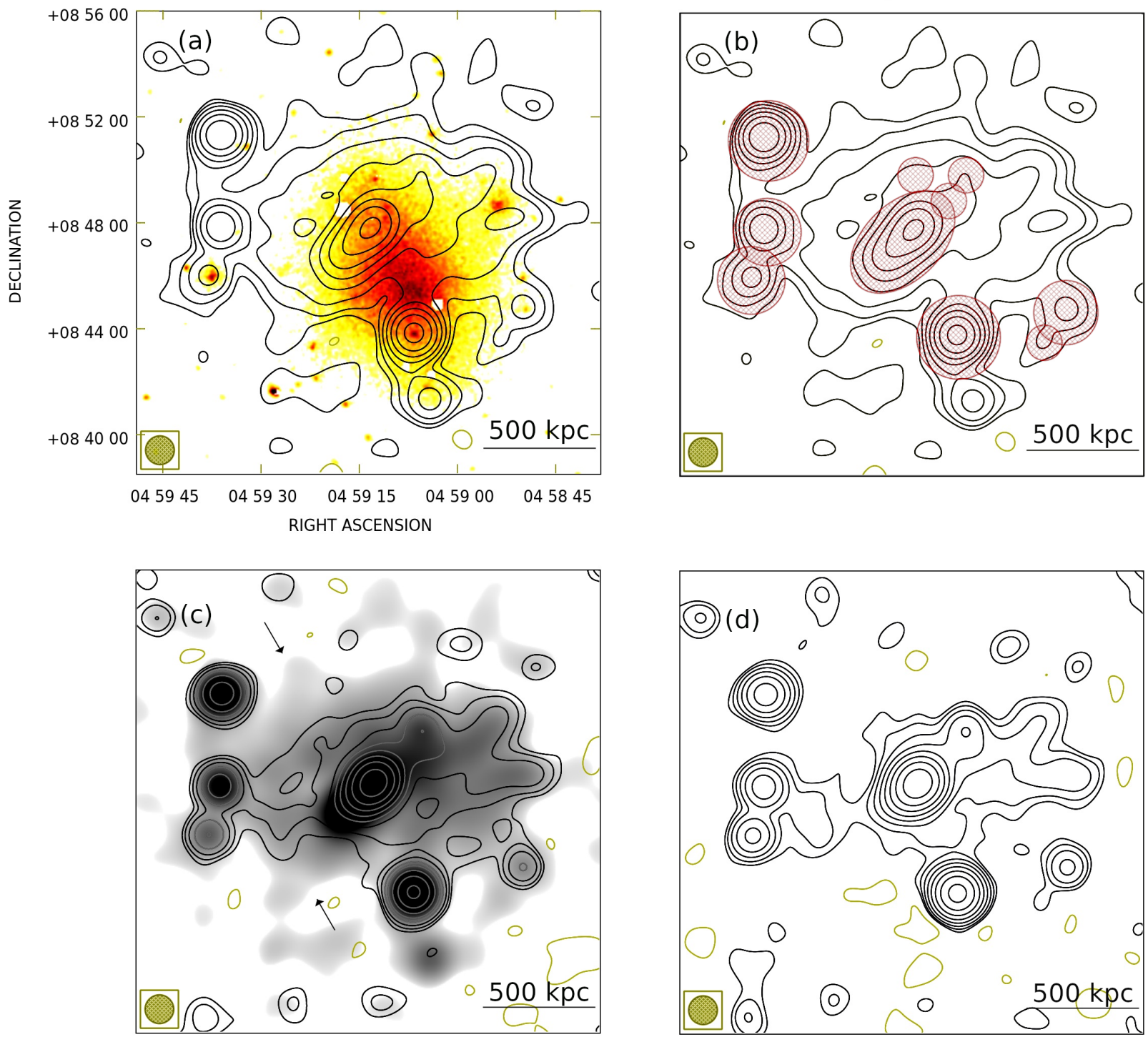

Figure 3. Panel (a): Radio emission in black contours from the galaxy cluster A523 at $144 \mathrm{MHz}(\sigma=1.3 \mathrm{mJy} / \mathrm{beam})$ overlaid on the X-ray image of the cluster from XMM-Newton (Cova et al. 2019) in colors, see the text for more details. Panel (b): Contours are the same as in panel (a), with regions used to mask the emission of discrete sources shown in red. Panel (c): Radio emission in black contours from the galaxy cluster A523 at $1.410 \mathrm{GHz}(\sigma=0.14 \mathrm{mJy} / \mathrm{beam})$ overlaid on the radio emission in gray colors at $144 \mathrm{MHz}$. Arrows point to the regions in the north-east and south-east detected with LOFAR but not with the VLA. Panel $(d)$ : Radio emission in black contours from the galaxy cluster A523 at $1.782 \mathrm{GHz}(\sigma=0.11 \mathrm{mJy} / \mathrm{beam})$. All radio images are shown at $65^{\prime \prime}$ of resolution and the synthesised beam is shown in the bottom left. Black contours start at $3 \sigma$ and scale by a factor 2 , while negative $3 \sigma$ contours are drawn in army green. In each panel the horizontal black bar corresponds to $500 \mathrm{kpc}$ at the chosen cosmology.

Table 4. Details of the spectral index maps. Col. 1, 2: minimum and maximum cut in $u v$-range; Col. 3, 4: minimum and maximum angular scales accessible with the data; Col. 5: original resolution of the $144 \mathrm{MHz}$ image; Col. 6: original resolution of the $1.410 \mathrm{GHz}$ image; Col. 7: restored beam.

\begin{tabular}{ccccccc}
\hline \hline $\begin{array}{c}u v-\min \\
\lambda\end{array}$ & $\begin{array}{c}u v \text {-max } \\
\lambda\end{array}$ & $\begin{array}{c}\theta_{\min } \\
\prime \prime\end{array}$ & $\begin{array}{c}\theta_{\max } \\
\prime\end{array}$ & $\begin{array}{c}\text { Beam } 144 \mathrm{MHz} \\
\prime \times \prime \prime\end{array}$ & $\begin{array}{c}\text { Beam } 1.410 \mathrm{GHz} \\
\| \times \prime \prime\end{array}$ & $\begin{array}{c}\text { Beam } \\
\| \times{ }_{\text {restored }}\end{array}$ \\
\hline 158 & 4835 & 48 & 22 & $34.5 \times 46.6$ & $36.1 \times 49.8$ & $65 \times 65$ \\
158 & 16980 & 14 & 22 & $7.8 \times 15.9$ & $13.0 \times 16.3$ & $20 \times 20$ \\
\hline \hline
\end{tabular}




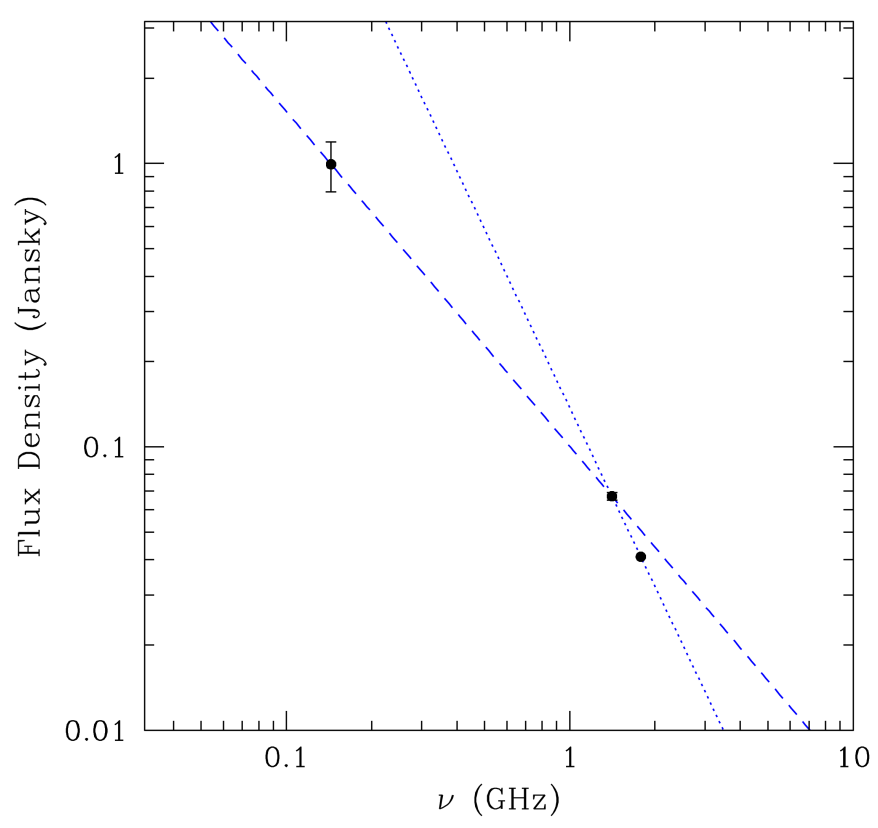

Figure 4. Flux density of the diffuse emission in A523 versus frequency. The power-law fit of the $144 \mathrm{MHz}$ and $1.410 \mathrm{GHz}$ data points is shown in dashed blue, while the fit of the $1.410 \mathrm{GHz}$ and $1.782 \mathrm{GHz}$ data points in dotted blue.

However, our frequency range is smaller than in these studies, where it extends up to about $5 \mathrm{GHz}$.

In order to perform a detailed analysis of the spectral index properties of the diffuse emission, we produced spectral index images at low- and high-spatial resolution with our new LOFAR observations at $144 \mathrm{MHz}$ and the VLA data at $1.410 \mathrm{GHz}$ presented in $\S 3$. We selected the same $u v$-range and weighting-scheme (robust=-0.5) at the two frequencies both at high and at low spatial resolution and a beam of $65^{\prime \prime}$ and $20^{\prime \prime}$ was restored, respectively, in the two cases. All the details about the selected $u v$-range, the corresponding angular scales accessible through the data, the original spatial resolution of the images and the size of restored beams are reported in Table 4. All the images have been regridded to a common frame in order to have the same pixel size.

The spectral index maps have been obtained by applying a sensitivity cut in radio brightness of $3 \sigma$ at both frequencies simultaneously, in order to exclude pixels that are below $3 \sigma$ in at least one of the two images. Flat-spectrum regions above the noise level at $1.410 \mathrm{GHz}$ but not at $144 \mathrm{MHz}$ and steep-spectrum regions detected at $144 \mathrm{MHz}$ but not at $1.410 \mathrm{GHz}$ will be missed with this procedure. In particular, the peripheral regions that are detected at $144 \mathrm{MHz}$ but not at $1.410 \mathrm{GHz}$ are characterized by a spectral index steeper than $\alpha \gtrsim 0.9$. In order to investigate the spectral index behaviour also in these regions, we directly compare the brightness profile at $144 \mathrm{MHz}$ and $1.410 \mathrm{GHz}$ without applying any sensitivity cut to the images.

\subsection{Spectral index images}

The spectral index distribution (left) and its uncertainty (right) are shown in Fig. 5 at both low (65", top panels) and high (20" , bottom panels) spatial resolution. The spectral index trends observed at low resolution are consistent with those observed at higher spatial resolution. However, at $20^{\prime \prime}$ resolution, only the brightest parts of the emission are visible.
Table 5. Spectral index values for the full diffuse emission, the filaments and along each filament. Col. 1: Region considered for the average; Col. 2, 3, 4: average spectral index $\langle\alpha\rangle$, its dispersion $\sigma_{\alpha}$ and the average uncertainty $\left\langle\sigma_{\alpha}\right\rangle$; Col. 5: spatial resolution of the images used for the measurements.

\begin{tabular}{|c|c|c|c|c|}
\hline Source & $\langle\alpha\rangle$ & $\sigma_{\alpha}$ & $\left\langle\sigma_{\alpha}\right\rangle$ & Beam ( $(\prime)$ \\
\hline All & 1.2 & 0.2 & 0.1 & 65 \\
\hline Filaments & 1.2 & 0.2 & 0.1 & 65 \\
\hline $\mathrm{F} 1$ & 1.3 & 0.2 & 0.1 & 65 \\
\hline F2 & 1.2 & 0.3 & 0.1 & 65 \\
\hline F3 & 1.2 & 0.2 & 0.1 & 65 \\
\hline Filaments & 1.0 & 0.2 & 0.1 & 20 \\
\hline $\mathrm{F} 1$ & 1.1 & 0.2 & 0.1 & 20 \\
\hline $\mathrm{F} 2$ & 0.9 & 0.1 & 0.2 & 20 \\
\hline
\end{tabular}

The low-resolution spectral index image between $144 \mathrm{MHz}$ and $1.410 \mathrm{GHz}$ reveals a patchy distribution with a flattening in the west at the location of the filaments, and a steepening both in the northeast and in the south-east. In particular, the region in the south-east of $\mathrm{S} 1$ is characterized by a spectral index $\alpha \sim 1.2-2.2$, likely because it is contaminated at least in part by the steep spectrum tails of S1. The north-east and south of the diffuse emission appear well detected in the $144 \mathrm{MHz}$ image but fall below the noise at $1.410 \mathrm{GHz}$, with the brightest patches at $144 \mathrm{MHz}$ reaching radio brightness up to $20 \mathrm{mJy} / \mathrm{beam}$. If we take a brightness upper limit of $0.39 \mathrm{mJy} / \mathrm{beam}$ at $1.410 \mathrm{GHz}$ (i.e., $3 \sigma$ with $\sigma=0.13 \mathrm{mJy} / \mathrm{beam}$ ), we derive a spectral index $\alpha>1.7$.

In Fig. 6 (panel (a)) we show the histogram of the spectral index values of the diffuse emission obtained covering the low resolution spectral index image with a grid, after masking compact sources, as shown in the inset. The side of the cells is equal to the full width at half maximum (FWHM) of the beam. We derive an average spectral index $\langle\alpha\rangle=1.2$, a dispersion $\sigma_{\alpha}=0.2$, and an average value of the spectral index statistical uncertainty $\left\langle\sigma_{\alpha}\right\rangle=0.1$, see Table $5^{6}$. The average spectral index statistical uncertainty has been derived from the spectral index uncertainty image (top right panel in Fig. 5). This image has been obtained by evaluating the statistical uncertainty on a pixel basis, including both the flux density scale uncertainty and the thermal noise of the two radio images. If the patchy structure of the spectral index image is due to measurement uncertainties, we expect that the mean value of the uncertainty image and the dispersion of the spectral index distribution are consistent. The dispersion is only slightly higher than the uncertainty derived by using the spectral index noise image suggesting that, even if some degree of intrinsic complexity is present, the observed fluctuations are dominated by the measurement uncertainties. Following Orrú et al. (2007), since the total dispersion is the result of the sum in quadrature of the uncertainty and of the intrinsic scatter

$\sigma_{\alpha}=\sqrt{\left\langle\sigma_{\alpha}\right\rangle^{2}+\sigma_{\text {int,proj }}^{2}}$,

we derive an intrinsic scatter in the spectral index $\sigma_{\text {int,proj }}=0.17$ projected in the plane of the sky on scales of the beam, i.e. $65^{\prime \prime}$. If the spectral index varies stochastically along the line of sight over the full diffuse emission region, we derive a 3-dimensional intrinsic spectral index $\sigma_{\text {int }}=\sqrt{N} \sigma_{\text {int,proj }}=0.67$, where $N=15$ is the number of cells assuming that the maximum size of the source along the line

6 Values derived by weighting the average radio brightness both at $144 \mathrm{MHz}$ and at $1.410 \mathrm{GHz}$ in the corresponding cell are in agreement with these values within the uncertainty. 

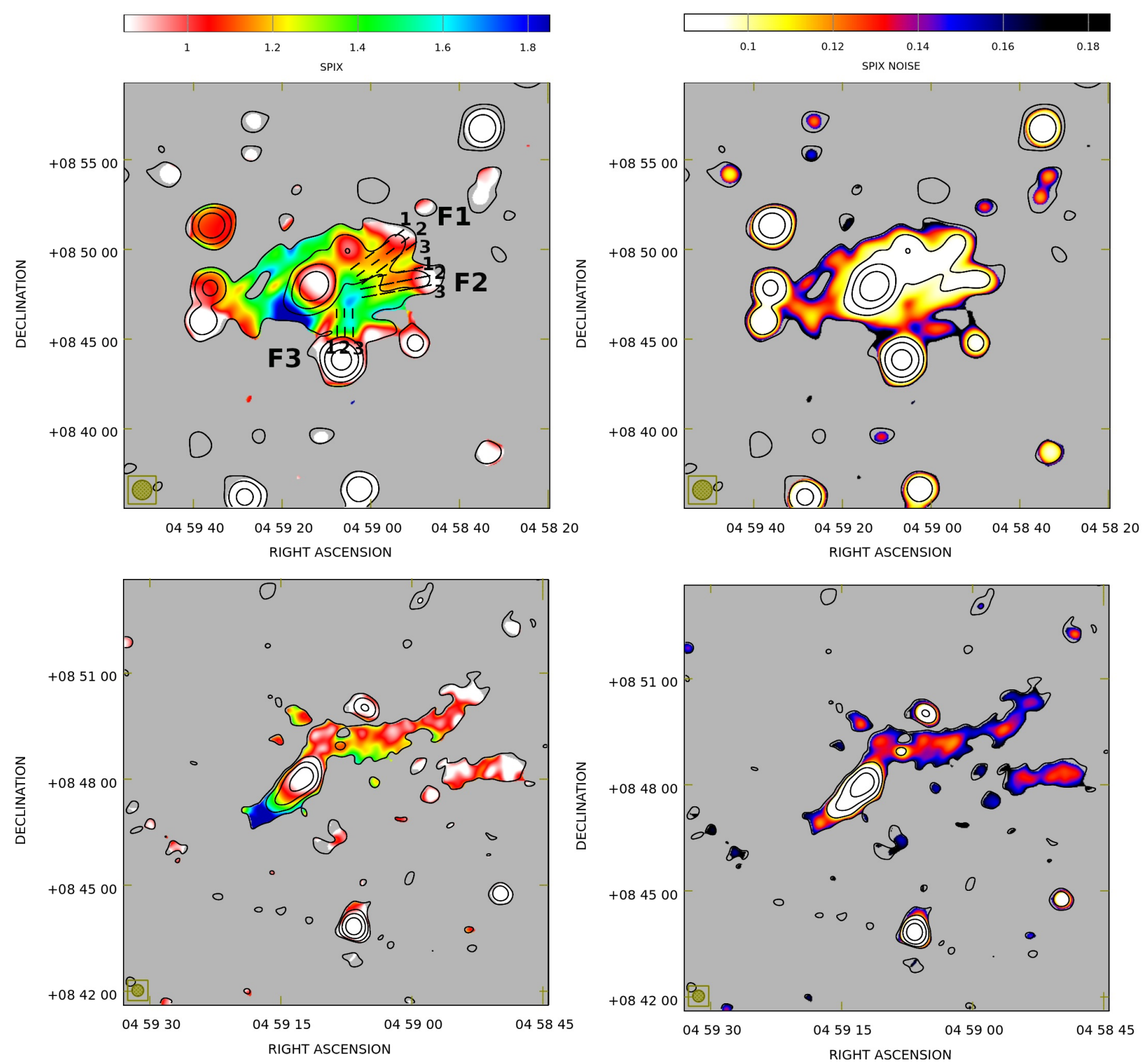

Figure 5. Top panels: Spectral index (left) and spectral index uncertainty (right) image of A523 between $144 \mathrm{MHz}$ and $1.410 \mathrm{GHz}$ at $65^{\prime \prime}$ resolution. Contours represent the radio emission at $1.410 \mathrm{GHz}$ at the same spatial resolution, start at $3 \sigma$ and increase by a factor $4(\sigma=0.13 \mathrm{mJy} / \mathrm{beam})$. Bottom panels: Spectral index (left) and spectral index uncertainty (right) image of the central region of A523 between $144 \mathrm{MHz}$ and $1.410 \mathrm{GHz}$ at $20^{\prime \prime}$ resolution. Contours represent the radio emission at $1.410 \mathrm{GHz}$ at the same spatial resolution, start at $3 \sigma$ and increase by a factor $4(\sigma=75 \mu \mathrm{Jy} / \mathrm{beam})$.

of sight is similar to the largest linear size in the plane of the sky, i.e., 15'. This is comparable to the value derived by Botteon et al. (2020) for the emission in A2255 and higher than the value derived for the radio halos in the Toothbrush cluster by van Weeren et al. (2016b) and in A520 by Hoang et al. (2019) by a factor of $\sim 7$ and 2 respectively.

\section{Filaments}

In the west of the source, the spectral index distribution shows a flattening at the location of the three filaments. Histograms of the spectral index distribution in this region are shown in the panels (b) and (c) of Fig. 6. In the central panel, we show the statistics derived from the low-resolution image, using the LOFAR image at $20^{\prime \prime}$ to determine the location and size of the filaments. Considering all the filaments simultaneously, the average spectral index is $\langle\alpha\rangle=$ $1.2 \pm 0.1$. When considered separately, we derive respectively $\langle\alpha\rangle_{\mathrm{F} 1}=$ $1.3 \pm 0.1,\langle\alpha\rangle_{\mathrm{F} 2}=1.2 \pm 0.1$, and $\langle\alpha\rangle_{\mathrm{F} 3}=1.2 \pm 0.1$, see Table 5 . However, the low resolution image hinders a detailed analysis of the spectral properties of the filaments, due to a blending with the rest of the diffuse emission. In the panel (c) of Fig. 6, we show the histograms of the spectral index distribution along the filaments obtained from the high-resolution image. We carried out our measurements only 

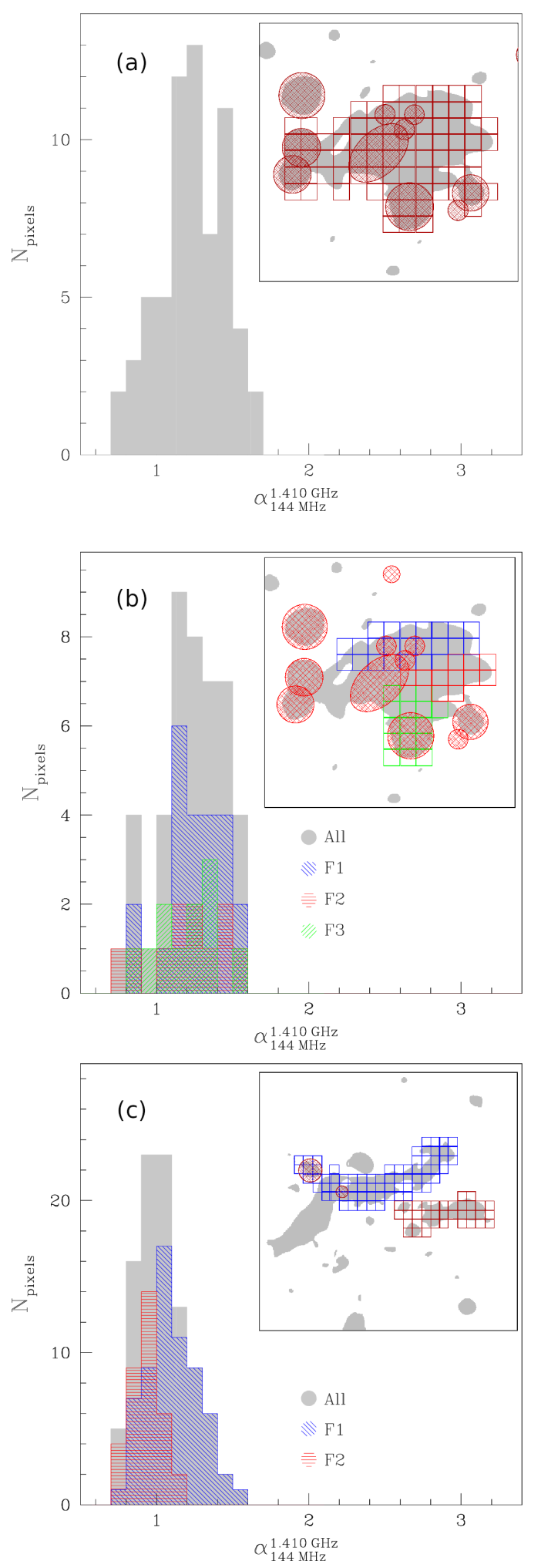

Figure 6. Panel (a): Histogram of the low-resolution spectral index image obtained covering the image with the grid shown in the inset and masking discrete sources. Panel (b): Histogram of the spectral index values at the location of the filaments at 65" (overall in gray, F1 only in blue, F2 only in red, and F3 only in green). Panel (c): Histogram of the spectral index values at the location of the filaments at $20^{\prime \prime}$ (overall in gray, F1 only in blue, and F2 only in red). In all cases the side of the cells of the grid has a size equal to the beam FWHM.

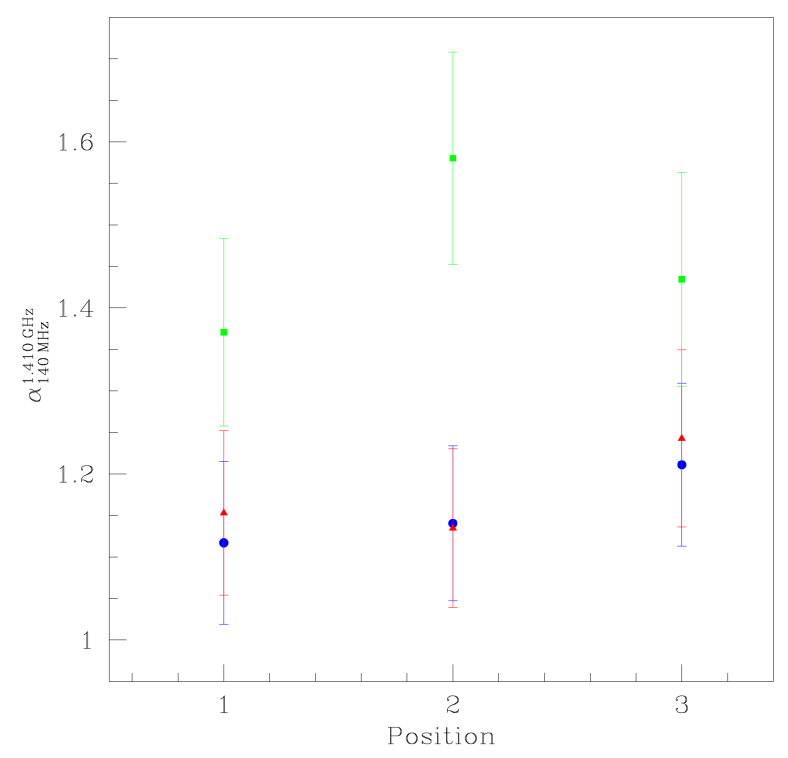

Figure 7. Spectral index along filament F1 (blue dots), filament F2 (red triangles) and filament F3 (green squares). The slices are shown in the top left panel of Fig. 5 and the numbers on the $\mathrm{x}$-axis indicate the slice identification number as reported in the same figure, see the text for more details.

for F1, still clearly visible, and for the brightest region of F2. Only a small patch of F3 survives, therefore we did not include it in the analysis. We find an overall average spectral index $\langle\alpha\rangle=1.0 \pm 0.1$, and $\langle\alpha\rangle_{\mathrm{F} 1}=1.1 \pm 0.1$ and $\langle\alpha\rangle_{\mathrm{F} 2}=0.9 \pm 0.2$, respectively for $\mathrm{F} 1$ and $\mathrm{F} 2$, see Table 5. Even if still in agreement within the measurement uncertainties, the spectral index in the brightest regions of these filaments appears slightly flatter than the rest of the source, and in particular, the emission along the filaments F2 is characterized by a flatter spectrum than that along F1. However, this difference could be due to the fact that in the high resolution image we are only probing the brightest regions of F2, while for F1 more structure is used.

A qualitative inspection of the $65^{\prime \prime}$ image indicates that moving away from the filament brightest region, the spectral index becomes steeper. To quantify this behaviour, we computed the spectral index along slices in the low-resolution image. For each filament we used three slices: a central one located along the brightest part of the filament and two others, one on each side. Slices are one pixel in width, spaced by half FWHM (i.e., 32.5") and with lengths comparable to the length of the filaments. To better identify the filaments we used the high resolution LOFAR image at $20^{\prime \prime}$. In Fig. 7, we show the average spectral index along the three slices for the three filaments: filament $\mathrm{F} 1$ in blue dots, filament F2 in red triangles and filament F3 in green boxes. The location of the slices is shown in Fig. 5 and is identified with the numbers 1, 2, and 3 (numbered from North to South for F1 and F2 and from left to right for F3), as reported in the $\mathrm{x}$-axis of Fig. 7. F1 shows a spectral index decreasing from south to north, while F2 shows a spectral index slightly steeper moving towards both north and south. However, within the errors, the two filaments show similar spectral indices with variations between 1 and 1.3. Finally, F3 has a steeper spectral index that varies in the range 1.3-1.7 with increasing values at the location of the brightest patches of the emission. The different spectral behaviour of F1 and F2 with respect to the surrounding medium could be related to a stronger magnetic field that allows us to detect lower-energy electrons with a flatter spectrum. Spectral index images of these filaments at higher 
resolution are necessary to investigate in more depth their behaviour, with no contamination from the rest of the diffuse emission.

Filaments of diffuse emission have also been observed in the galaxy cluster A2255. Govoni et al. (2005) classified them as peripheral structures associated with the radio halo in agreement with expectations from numerical simulations (Loi et al. 2019), while Pizzo et al. (2011) classified them as relics, due to their morphology, fractional polarization level and rotation measure. The filaments in A2255 show spectral indices flatter $(\alpha \sim 0.8-1.3)$ than the rest of the diffuse emission (up to $\alpha \sim 2$ ), see also Botteon et al. (2020). Similar filamentary emission has been recently identified in the diffuse emission in MACS J0717.5+3745 characterized by a spectral index of $\alpha \sim 1.2$ (Rajpurohit et al. 2021a). Rajpurohit et al. (2021b) speculate that such features may be due to the complex distributions of shocks caused by the merger in the cluster. In line with this, the filaments in A523 could be either radio relics or associated with the peripheral regions of the radio halo. Discriminating between the two is not a trivial task. F1 and F2 could be associated with shock waves produced by the main merger along the SSW-NNE direction, while F3 could be associated with a shock wave produced by the secondary merger along the perpendicular axis. Shock fronts have not been detected at the sensitivity of X-ray observations currently available and, only in case of the filament $\mathrm{F} 1$, the morphology and the spectral index distribution could support this possibility. In the case of a merger with some inclination with respect to the plane of the sky, the detection of shock waves and of a spectral gradient could be hindered by projection effects. The available X-ray and optical data do not show evidence of a merger component along the line of sight. Such a component could be possibly associated with the secondary merger. However, at the moment, no indication of this emerges from the data. For a detailed description of the sub-clumps and of the merger geometry see Girardi et al. (2016) and Cova et al. (2019).

\section{Patch $P$}

Patch P is clearly detected at $144 \mathrm{MHz}$ at about RA $04 \mathrm{~h}: 59 \mathrm{~m}: 04 \mathrm{~s}$ and Dec $+08^{\circ}: 41^{\prime}: 23^{\prime \prime}$, while only the peak of the emission associated with it is visible at $1.410 \mathrm{GHz}$, with a brightness of $\approx 0.4 \mathrm{mJy} / \mathrm{beam}$. If we compare this value with the peak radio brightness at $144 \mathrm{MHz}$, i.e. $42 \mathrm{mJy} /$ beam, we obtain a spectral index $\alpha=2.0$ in the brightest region of the emission. This steep value might suggest that the patch $\mathrm{P}$ could be a very old object, relic emission from a radio lobe, or plasma emitted by a now-quiescent AGN. The closest radio galaxy is the source $S 5(\mathrm{z}=0.1041$, Girardi et al. 2016) which shows signs of possible re-starting jets when inspected at high spatial resolution (see Fig. 1). Patch $\mathrm{P}$ is distant almost $\sim 2.5^{\prime}$ from S5, corresponding to a projected linear distance of about $300 \mathrm{kpc}$. If patch $\mathrm{P}$ is a remnant lobe of previous activity of S5, it could have moved up to the distance where we observe it now. In this case, its linear size would be about $170 \mathrm{kpc}$. Alternatively, if we select close optical sources from Girardi et al. (2016) and Golovich et al. (2019) within a radial distance of one beam $\left(65^{\prime \prime}\right)$ from the peak of patch $\mathrm{P}$, we find 18 sources with redshift $0.097 \lesssim z \lesssim 0.637$, translating into a linear size of the source between $170 \mathrm{kpc}$ and $640 \mathrm{kpc}^{7}$, the closest being a source at $\mathrm{z} \approx 0.637$. According to radiative cooling models, old remnant sources are expected to be characterized by very steep spectra ( $\alpha \gtrsim 1.2$, Pacholczyk 1970) and observations of the Lockman Hole field with LOFAR show that these sources represent a few percent of

\footnotetext{
7 We measure a largest angular size of $\sim 1.5^{\prime}$ in Fig. 2
}

the low resolution catalogue of radio sources in this field (Brienza et al. 2017).

A second possibility is that this source is the continuation of the radio filament F3 or a diffuse synchrotron emission associated with an high-redshift galaxy cluster, since the closest galaxy in projection is the galaxy at $\mathrm{z}=0.637$ detected at $\approx 11^{\prime \prime}$ from the source radio peak. In the latter case the radio largest linear size of the source would be about $570 \mathrm{kpc}$, its radio power ${ }^{8}$ at $144 \mathrm{MHz} \sim 1.7 \times 10^{26} \mathrm{~W} / \mathrm{Hz}$ and at $1.410 \mathrm{GHz} \sim 1.8 \times 10^{24} \mathrm{~W} / \mathrm{Hz}$, still consistent with typical radio powers observed for radio halos (see Giovannini et al. 2009, Feretti et al. 2012 and van Weeren et al. 2019). Assuming the scaling relation $P_{150 \mathrm{MHz}}-M_{\mathrm{SZ}, 500}, P_{1.4 \mathrm{GHz}^{-}} M_{\mathrm{X}, 500}$, and $P_{1.4 \mathrm{GHz}^{-}}$

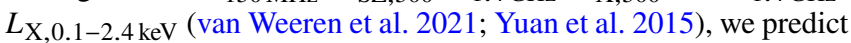
a mass of the system $M_{500}$ in the range $8-11 \times 10^{14} M_{\odot}$ and an Xray luminosity $L_{\mathrm{X}, 0.1-2.4 \mathrm{keV}}=4-18 \times 10^{44} M_{\odot}$. These values are consistent with those observed for galaxy clusters at similar redshift (e.g., Schneider 2015). However, we note that such a system would be very massive and therefore very rare.

Finally, a third possibility is that this source is an Odd Radio Circle (ORC, Norris et al. 2021). The size, morphology and spectral properties resemble those of this new class of sources discovered with Australian Square Kilometre Array Pathfinder (ASKAP). The known ORCs have been observed in the direction of high-redshift galaxies and, interestingly, close to the location of patch $\mathrm{P}$ one galaxy is detected at $\mathrm{z}=0.637$. However, these objects show a bright limb not observed here and the galactic latitude of patch $\mathrm{P}$ is lower than that observed for ORCs. If confirmed, this would be the first ORC seen by LOFAR.

\subsection{Spectral index profile}

In Fig. 8, the radial profiles of the radio brightness at $144 \mathrm{MHz}$ (black dots) and $1.410 \mathrm{GHz}$ (blue triangles) are shown in panel (a), while the corresponding spectral index profile is shown in panel (b). These profiles are derived by using concentric annuli centered on the radio peak at $144 \mathrm{MHz}$ on the same $65^{\prime \prime}$ radio brightness images used to derive the spectral index spatial distribution. The annuli have widths of one beam size. The statistics are evaluated after masking all the embedded discrete sources as shown in the right panel, including S1, whose steep tails could otherwise contaminate our estimation. A few additional sources have been masked with respect to those labeled in Fig. 1 because they are characterized by a radio brightness peak $\gtrsim 8 \sigma$ at $1.410 \mathrm{GHz}$.

Panel (c) in Fig. 8 shows the pixel distribution of the spectral index image at $65^{\prime \prime}$ (top left panel in Fig. 5), as a function of the distance from the radio peak at $144 \mathrm{MHz}$, after masking embedded discrete sources. The pixel distribution indicates that the low-resolution image only properly samples regions out to $500-600 \mathrm{kpc}$ due to the sensitivity cut we applied. In this region, the radial profile provides an average spectral index $\alpha \sim 1.2$, in agreement within the uncertainty with the mean over the spectral index image, see $\S 5.1$. Since we do not apply any sensitivity cut to derive the spectral index radial profile, the radial profile can give us some indication about the spectral index behaviour also beyond $600 \mathrm{kpc}$, in regions where the radio brightness is below the $3 \sigma$ level. In this way, possible flattening and/or steepening in the spectral index distribution can be possibly detected. Beyond $600 \mathrm{kpc}$, we derive a lower limit of the spectral index of $\alpha \gtrsim 1.5$. Results in regions covered by the most external

8 We assumed a spectral index $\alpha=2$ as derived for the brightest region of the emission, see text above. 


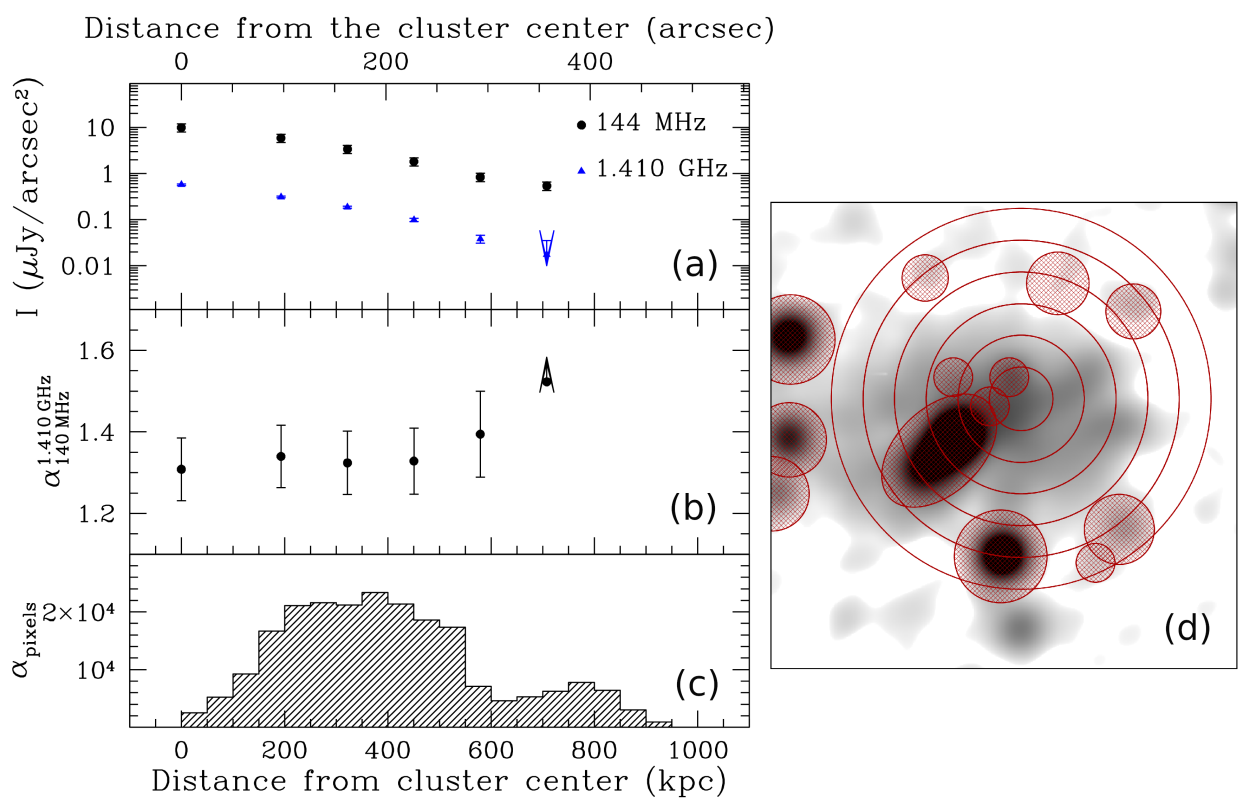

Figure 8. Panel (a): Radial profile of the radio brightness at $144 \mathrm{MHz}$ (black dots) and $1.410 \mathrm{GHz}$ (blue triangles) with respect to the radio peak, using the annuli shown in the panel (d). Panel (b): Radial profile of the spectral index of the diffuse emission obtained from the radio brightness profiles in the panel (a). Panel (c): pixel distribution of the spectral index $65^{\prime \prime}$ image as a function of the distance from the radio peak at $144 \mathrm{MHz}$. The pixel distribution does not depend on the annuli chosen to produce the radial profiles shown in the above panels. Panel (d): Gray scale shows the LOFAR image at $144 \mathrm{MHz}$ at $65^{\prime \prime}$, as well as annuli with a beam-size width centered on the radio peak at $144 \mathrm{MHz}$ and used in order to derive the radial profile. Discrete sources have been masked and therefore not included in the measurements.

annulus must be interpreted with caution. Indeed, the emission here is below the noise level of an individual beam and, as we used a deconvolution threshold of $1 \sigma$ and therefore signal below this threshold it is not deconvolved, LOFAR emission may result in a brighter signal than reality, leading to artificial spectral steepening. Moreover, we note that even though we match VLA and LOFAR $u v$-range, the density of the points in the inner of the $u v$-plane is much higher in LOFAR than in the VLA data. However, the impact of this on the images is very difficult to estimate. The diffuse emission in A523 does not show a circular shape and the radio and X-ray emission do not fully overlap. A substantial offset between the peak in X-ray surface brightness and radio brightness is also present at $144 \mathrm{MHz}$, the largest absolute offset observed for radio halos and among the largest fractional offsets (offset/size) when giant radio halos only are considered (Feretti et al. 2012), as expected in case of a magnetic field fluctuating on large spatial scales (e.g., Vacca et al. 2010). This offset makes the radial profile of the spectral index and its link with the thermal properties of the system difficult to interpret. No steepening is observed even when the spectral index is computed as a function of the distance from the peak in the X-ray emission (not shown here).

\subsection{Spectral index of discrete sources}

The presence of the radio galaxies identified in $\S 4$ is apparent in the spectral index images shown in Fig. 5. Flux densities of these sources have been derived as described in $\S 4$. Spectral index values between $144 \mathrm{MHz} 1.410 \mathrm{GHz}$ are given in Table 3 . For the sake of comparison, we report in Table 3 the flux density and spectral index values by de Gasperin et al. (2018), derived by using observations at $147 \mathrm{MHz}$ from the TIFR GMRT Sky Survey (TGSS, Intema et al. 2017) and at $1.4 \mathrm{GHz}$ from the NVSS (Condon et al. 1998). The sources in the catalog have been identified by imposing a distance in RA and Dec respectively of $\lesssim 25^{\prime \prime}$ from our sources, comparable to the TGSS spatial resolution. TGSS flux densities are available for four out of ten sources and the NVSS flux densities for six of them. The remaining sources are probably too faint to be detected at the corresponding sensitivity of TGSS and/or NVSS. The NVSS fux density of the sources agrees with our VLA values within at most $1.3 \sigma$, while the TGSS measurements agree with our LOFAR flux densities within at most $1.9 \sigma$, with LOFAR measurements being systematically slightly higher, ${ }^{9}$. This is likely due to a difference in the flux density scale of our LOFAR observations with respect to the TGSS data that, comparing the flux densities of these four sources, amounts to a factor of about $1.4-1.6$. Position-dependent flux density scale variations have been found in the TGSS by other authors (Hurley-Walker 2017). The de Gasperin et al. (2018) catalog reports spectral index information for six of our sources (S1, S5, S6, S7, S8, and S9, Col. 10, Table 3), derived from the TGSS and NVSS flux density values. However, for two of them (S6 and S9), the TGSS flux density given in the catalog is zero (see Col.6, Table 3), therefore it is unclear how the spectral index has been derived and we will neglect them in the following. The spectral indices of the remaining four sources differ with respect to our by about $2.3 \sigma$ for $\mathrm{S} 1,1.7 \sigma$ for S5, $0.4 \sigma$ for S7, and $1.5 \sigma$ for S8 (for each source $\sigma$ has been computed with the same approach described in Footnote 9). Our flux density measurements for $\mathrm{S} 1$ include the contribution from the diffuse emission. Given the size of the source and the mean radio brightness of the overall diffuse emission, this term amounts to about $35 \mathrm{mJy}$ and $2 \mathrm{mJy}$ respectively at $144 \mathrm{MHz}$ and $1.4 \mathrm{GHz}$. However, as per our knowledge, the flux densities given in the public catalog

9 The displacement $\sigma$ has been computed as $\left|S_{\mathrm{XX}}-S_{\mathrm{YY}}\right| / \sqrt{E_{\mathrm{XX}}^{2}+E_{\mathrm{YY}}^{2}}$, where $E_{\mathrm{XX}}$ and $E_{\mathrm{YY}}$ are the uncertainties in $S_{\mathrm{XX}}$ and $S_{\mathrm{YY}}$, as given in Table 3. 


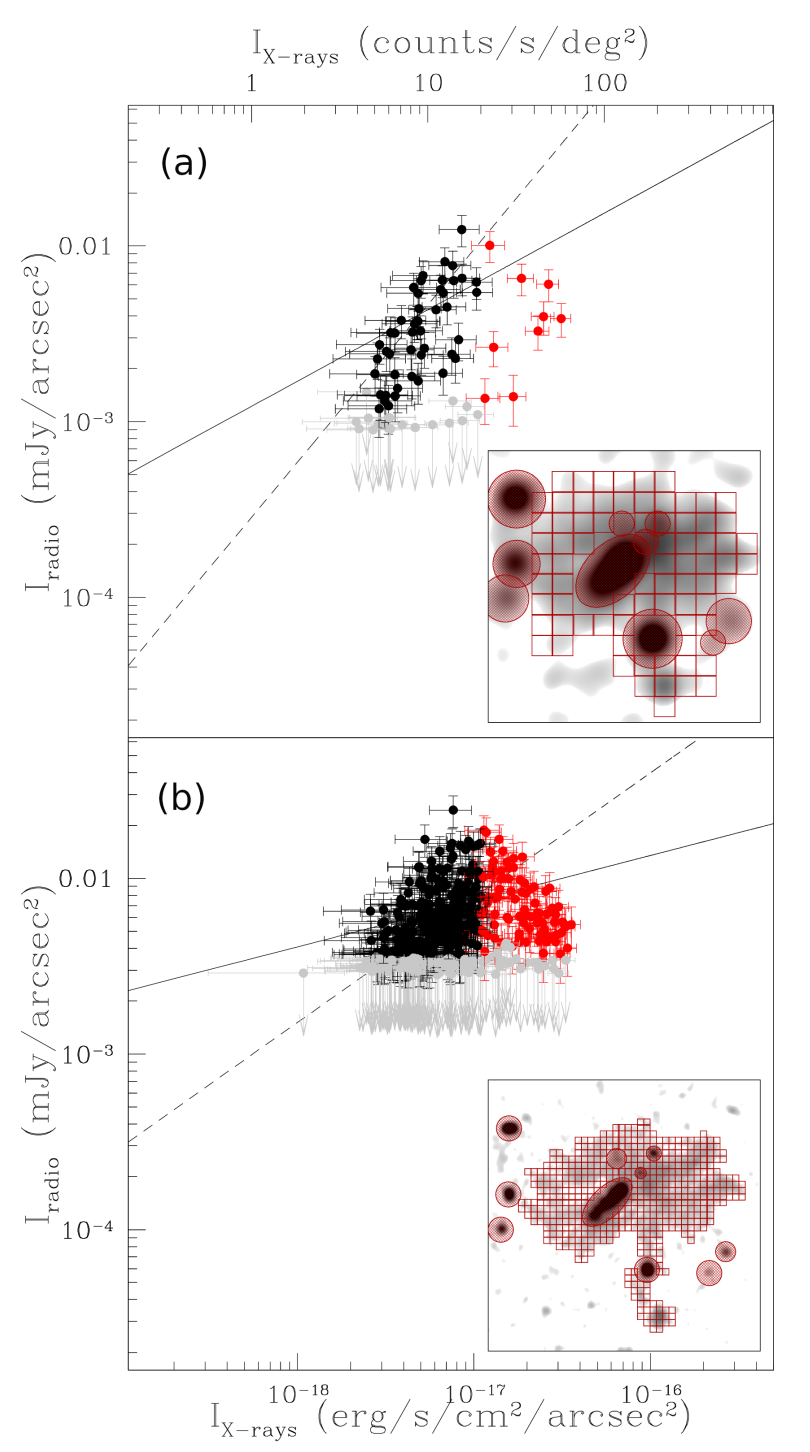

Figure 9. Radio $144 \mathrm{MHz}$ versus X-ray surface brightness of the cluster at $65^{\prime \prime}$ (panel (a)) and at 20" (panel (b)): upper limits of the radio emission in gray, points corresponding to X-ray emission larger than 20 counts/s/deg ${ }^{2}$ in red and smaller than 20 counts/s/deg ${ }^{2}$ in black. The continuous line represents the power-law fit including all data points, while the dashed line represents the fit only considering points corresponding to an X-ray emission lower than 20 counts/s/deg ${ }^{2}$ (including upper limits). The inset panels show the grid on the radio emission of the cluster at $144 \mathrm{MHz}$.

include this contribution as well and therefore the difference in the flux densities and in the spectral indices can not be ascribed to it. Our spectral index for source S1 is flatter than the published value, likely also because of the slightly higher flux density measured from the NVSS with respect to our VLA measurement. For the sources S7 and $\mathrm{S} 5$, the flux densities at $1.4 \mathrm{GHz}$ appear to be consistent within $2 \sigma$, therefore the difference in the spectral index is entirely due to our higher flux density at $144 \mathrm{MHz}$.

\section{COMPARISON WITH CLUSTER X-RAY PROPERTIES}

In this section we compare the radio properties of the cluster with the mass, X-ray emission, temperature, pressure, and entropy, looking for correlations among them as they contain information on the thermodynamic history of the system (see, e.g., Voit et al. 2005; Shi et al. 2020). To this end, we used the X-ray images presented by Cova et al. (2019).

\subsection{Local Radio - X-ray correlation}

Cova et al. (2019) compared the X-ray emission of the system with the radio properties at $1.4 \mathrm{GHz}$ by Girardi et al. (2016). Their results confirm that this radio halo is one of the most significant outliers with respect to the X-ray - radio correlation for radio halos (see also Giovannini et al. 2011). Moreover, they find that the X-ray and radio brightness at $1.4 \mathrm{GHz}$ do not show a point-to-point correlation. Here, we investigate the local radio - X-ray correlation using the data at $144 \mathrm{MHz}$.

In Fig. 9, we compare the X-ray brightness in the $0.5-2.5 \mathrm{keV}$ energy band with the radio brightness at $144 \mathrm{MHz}$, by using the images at $65^{\prime \prime}$ (panel (a)) and at 20" (panel (b)) spatial resolution. Compact sources have been masked and the diffuse emission has been covered using a grid with a cell-size equal to the beam FWHM, as shown in the inset. We show points corresponding to an X-ray signal $<20$ counts/s/deg ${ }^{2}$ in black, points corresponding to an X-ray signal $>20$ counts/s/deg ${ }^{2}$ in red, with radio upper limits in gray. We measured the radio versus X-ray surface brightness for each cell and, in order to deal with physical units, we converted it from counts $/ \mathrm{s} / \mathrm{deg}^{2}$ to $\mathrm{erg} / \mathrm{s} / \mathrm{cm}^{2} / \mathrm{arcsec}^{2}$, using the neutral hydrogen column density derived from HI4PI Survey (HI4PI Collaboration et al. 2016), the XMM/MOS Thin Count rate conversion factor (this detector is indeed used as a reference for deriving the scaling factor of the other detectors), and an APEC model mean temperature of $4.3 \mathrm{keV}$ and abundance of 0.2 the solar one obtained for A523 in Cova et al. (2019). Then we fitted in logarithmic scale a power-law of the form $I_{\text {radio }}=b\left(I_{\mathrm{X}-\text { rays }}\right)^{a}$ to the distribution, including all the data, with the scipy.optimize python package. At $65^{\prime \prime}$, we find $a=0.55 \pm 0.12$ and $\lg (b)=(7.13 \pm 2.12)$ with a Pearson correlation coefficient (Pearson 1895) $r_{\mathrm{P}}=0.41$ and a Spearman correlation coefficient (Spearman 1904; Myers 2003) $r_{\mathrm{S}}=0.48$, while at 20" $a=0.26 \pm 0.04$ and $\lg (b)=(2.29 \pm 0.70)$ with $r_{\mathrm{P}}=0.27$ and $r_{\mathrm{S}}=0.36$. Despite the weak correlation, it is not so flat as at $1.4 \mathrm{GHz}$ $\left(a=0.095 \pm 0.08, r_{\mathrm{S}}=0.27, r_{\mathrm{P}}=0.28\right.$, Cova et al. 2019).

If we progressively exclude from the fit points at high X-ray brightness, the correlation becomes steeper and steeper, and slightly stronger. The high resolution radio image provides better statistics and, in this case, the Pearson and Spearman coefficients reach both their maximum value $\left(r_{\mathrm{P}}=0.38\right.$ and $\left.r_{\mathrm{S}}=0.42\right)$ for an X-ray signal $<20$ counts/s/deg ${ }^{2}$. When we select only points corresponding to an X-ray signal $<20$ counts $/ \mathrm{s} / \mathrm{deg}^{2}$, we find $a=0.71 \pm 0.07$ and $\lg (b)=(9.96 \pm 1.21)$. In Fig. 9, we show the two fits: the continuous line includes all data points, while the dashed line only points with an X-ray signal $<20$ counts/s/deg ${ }^{2}$. In both cases, upper limits are taken into account. In Table 6, we report the values of $a, \lg (b)$ by the fitting routine, and the corresponding Pearson and Spearman coefficients when fitting points corresponding to different ranges of X-ray counts/s/deg ${ }^{2}$. At $65^{\prime \prime}$ the statistics quality is poor but includes regions of faint radio emission. At $20^{\prime \prime}$ we have more cells and we are able to better investigate the behaviour of bright patches that are averaged down by fainter surrounding regions at lower resolution. Results obtained excluding strong X-ray emission regions, typically coinciding with the cluster center, indicate that two trends are present: one flatter including all data points and one steeper including only faint X-ray emission. For the radio halo in A2255, Botteon et al. (2020) showed that higher thresholds in $I_{\text {radio }}$ translate to flatter slopes of the $I_{\text {radio }}-I_{\mathrm{X} \text {-rays }}$. Close to the threshold, the selection tends to pick 
Table 6. Results from the fit of the radio $144 \mathrm{MHz}$ brightness versus X-ray emission for regions characterised by an X-ray counts/s/deg ${ }^{2}$ given in Col. 1 . In Col. 2, 3 we report $a$ and $\lg (b)$, while in Col. 4, 5 the Pearson $r_{\mathrm{P}}$ and Spearman $r_{\mathrm{S}}$ coefficients. In Col. 6 the resolution of the radio image is given.

\begin{tabular}{cccccc}
\hline \hline X-rays counts/s/deg & $a$ & $l g(b)$ & $r_{\mathrm{P}}$ & $r_{\mathrm{S}}$ & Resolution $(")$ \\
\hline \hline $0-10$ & $0.93 \pm 0.16$ & $13.91 \pm 2.80$ & 0.36 & 0.29 & 20 \\
$0-20$ & $0.71 \pm 0.07$ & $9.96 \pm 1.21$ & 0.38 & 0.42 & 20 \\
$0-30$ & $0.61 \pm 0.06$ & $8.31 \pm 0.95$ & 0.36 & 0.41 & 20 \\
$0-40$ & $0.48 \pm 0.05$ & $6.09 \pm 0.84$ & 0.36 & 0.41 & 20 \\
$0-50$ & $0.35 \pm 0.04$ & $3.78 \pm 0.76$ & 0.32 & 0.38 & 20 \\
$0-60$ & $0.28 \pm 0.04$ & $2.66 \pm 0.72$ & 0.28 & 0.36 & 20 \\
$0-70$ & $0.26 \pm 0.04$ & $2.29 \pm 0.70$ & 0.27 & 0.36 & 20 \\
$0-30$ & $1.06 \pm 0.16$ & $15.89 \pm 2.81$ & 0.41 & 0.45 & 65 \\
$0-70$ & $0.55 \pm 0.12$ & $7.13 \pm 2.12$ & 0.41 & 0.48 & 65 \\
\hline \hline
\end{tabular}

up only the up-scattered values, with a consequent underestimate of the slope of the correlation in faint X-rays regions. Here, we are possibly observing a similar behaviour, when applying a selection on the $\mathrm{X}$-rays instead than in the radio brightness. However, in our case, two trends can be qualitatively distinguished in the plots in Fig. 9 (black and red points) even without applying any cut.

The behaviour observed in A523 differs from what observed for other clusters, i.e., A520 (Govoni et al. 2001b; Hoang et al. 2019) and 1E 0657-55.8 (Shimwell et al. 2014), which appear to be characterized by respectively an almost flat correlation or by not significant correlation at all. Indeed, a steeper sub-linear correlation emerges when brighter X-ray regions are excluded from statistics, although this correlation is weak. The behaviour observed in this system could reflect the complexity of the dynamical state of the system. The cluster is undergoing a primary merger along the SSW-NNE direction (Girardi et al. 2016; Golovich et al. 2019) and likely a secondary merger along the ESE-WNW direction (Cova et al. 2019). The flat component, weaker in radio, could be related to the primary merger brighter in X-rays. Our LOFAR data show a faint emission emerging in the north-east and in the south of the system, completely buried by the noise at higher frequencies. The steep component corresponds to regions stronger in radio and less bright in X-rays, being likely dominated by the emission in the north of the system. This structure is co-spatial with the possible secondary merger and the higher radio brightness associated with it could be the result of the superposition of the two mergers at this location. While the radio plasma along the SSW-NNE direction is accelerated only by the primary merger and only visible at LOFAR frequencies likely due to ageing, the radio plasma in the perpendicular direction gains energy also through the secondary merger, likely explaining the observed higher radio brightness.

\subsection{Radio emission and cluster mass}

The mass of clusters hosting radio halos correlates with the radio-halo power at $1.4 \mathrm{GHz}$ (e.g., Yuan et al. 2015), and at $150 \mathrm{MHz}$ (e.g., van Weeren et al. 2021). Numerical simulations show that the SZ effect is an excellent proxy of the cluster mass, with an intrinsic scatter of $\lesssim 10 \%$ (e.g., Nagai 2006), even if recent observations found a scatter up to $\sim 13 \%$ that can be significantly reduced when nonthermal pressure associated with ICM turbulent motions are taken into account (e.g., Yu et al. 2015). Galaxy cluster masses derived from X-ray observations can be affected by the dynamical state of the system. The cluster might be in the adiabatic expansion phase and, in this case, X-ray proxies such as luminosity and temperature could be lower than during the initial state, causing an underestimate of the mass of the system (see e.g., Ricker \& Sarazin 2001) with a bias up to $\sim 30 \%$ (e.g., Nagai et al. 2007). No SZ mass estimate is available for A523 and the X-ray derived mass is $M_{\mathrm{X}, 500}=2.2-3.6 \times 10^{14} M_{\odot}$. Considering our flux measurements at $1.410 \mathrm{GHz}$ and $144 \mathrm{MHz}$, taking into account the different cosmology, the frequency scaling when appropriate, and k-correction ${ }^{10}$, the value predicted according to the $P_{1.4 \mathrm{GHz}}-M_{\mathrm{X}, 500}$ scaling relation in Yuan et al. (2015) is $M_{500} \approx 6-10 \times 10^{14} M_{\odot}$, while $M_{500} \approx 6-9 \times 10^{14} M_{\odot}$ from the best fit relations $P_{150 \mathrm{GHz}}-M_{\mathrm{SZ}, 500}$ by van Weeren et al. (2021).

The values derived are larger than the mass obtained from X-ray observations. Concerning the estimate from $144 \mathrm{MHz}$ data and the van Weeren et al. (2021) scaling relation, we stress that the scaling relation is based on SZ data, while the mass estimate we are using here is derived from X-rays. If we assume that the observed X-ray mass is underestimated by about $30 \%$, we expect a mass of the system of $M_{500} \sim 5 \times 10^{14} M_{\odot}$, still lower than the value obtained from the scaling relation. In all these derivations the uncertainties both in the scaling relations and in our radio powers have been taken into account.

Based on $\sigma_{\mathrm{v}}$-mass scaling relations from optical data, Girardi et al. (2016) derive a cluster mass $M_{500}=5-6 \times 10^{14} M_{\odot}$ that is in better agreement with the values predicted by the radio power of the diffuse emission if this is a radio halo. We note that the uncertainty of the optical mass estimate varies between $7 \%$ and $30 \%$.

Overall, we find that the source remains outside the observed correlation both at $1.4 \mathrm{GHz}$ and $144 \mathrm{MHz}$. However, we note that the scaling relation is not well constrained in the mass range of A523, because most of present studies rely on high mass clusters. We need therefore to extrapolate the correlation in a poorly sampled region of the radio brightness-mass diagram. Moreover, the emission we observe in A523 could be the result of a superposition of different sources (diffuse emission associated with the two merger processes, but also the patch $\mathrm{P}$ and the filaments F1, F2 and F3, which may not belong to the radio halo). An estimation of the flux density associated with the filaments is very difficult because these structures are embedded in the diffuse large scale emission.

\subsection{Radio brightness versus temperature, entropy and pressure}

We compared the radio brightness distribution at $144 \mathrm{MHz}$ with the temperature $T$, pressure $P$ and pseudo-entropy $s$ images of the thermal gas by Cova et al. (2019). As stated in that paper, temperatures

10 A spectral index $\alpha=1.2 \pm 0.2$ has been assumed, please refer to $§ 5.1$. 

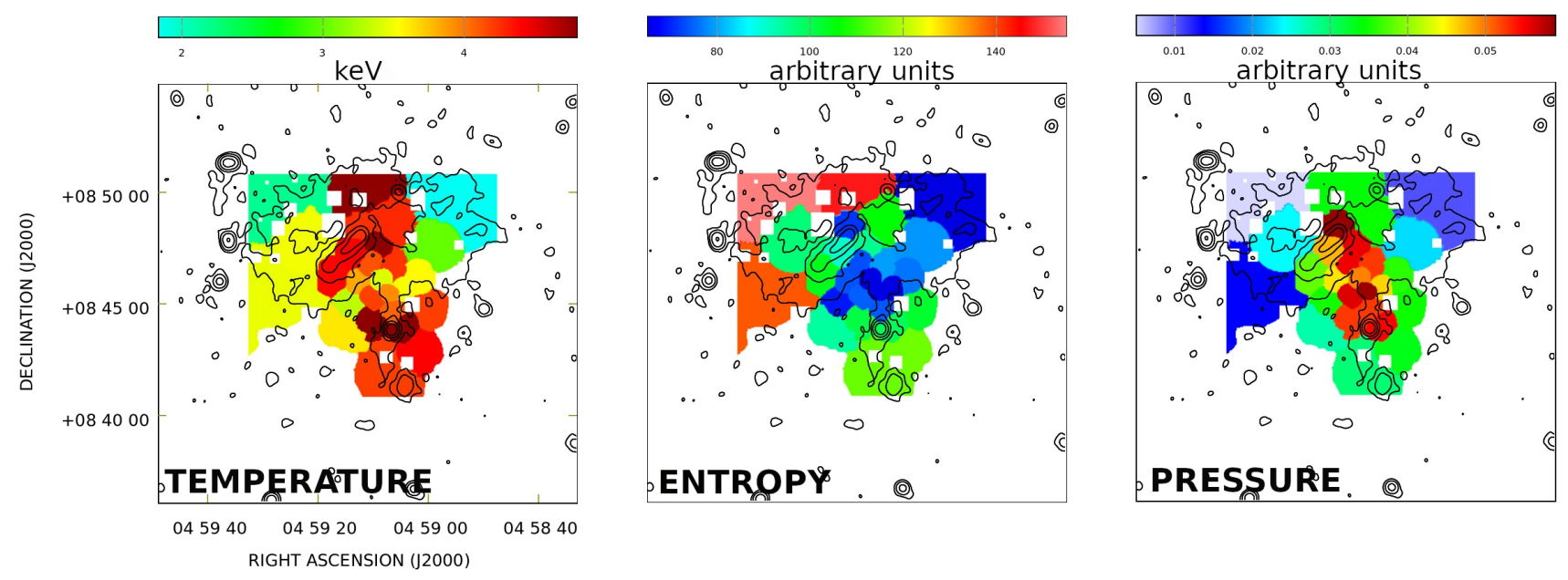

Figure 10. Temperature (left panel), entropy (middle panel), and pressure (right panel) images in colors, taken from Cova et al. (2019). While temperature is shown in $\mathrm{keV}$, entropy and pressure are shown in arbitrary units. Contours represent the radio brightness at $144 \mathrm{MHz}$ at $20^{\prime \prime}$, start at $3 \sigma(\sigma=0.4 \mathrm{mJy} / \mathrm{beam})$ and increase by a factor 4 . They have been derived from the LOFAR images presented in this work. The field of view is the same in all the images.

$T$ are directly derived through the spectral fitting, while the pressure $P$ and the entropy $s$ are calculated following Rossetti et al. (2007)

$P=T \times \mathrm{EM}^{1 / 2} \mathrm{keV} \mathrm{cm}^{-5 / 2} \operatorname{arcmin}^{-1}$

and

$s=T \times \mathrm{EM}^{-1 / 3} \mathrm{keV} \mathrm{cm}^{5 / 3} \operatorname{arcmin}^{-2 / 3}$,

where EM is the projected emission measure. Both pressure and entropy values are pseudo quantities, meaning that they are projected along the line of sight.

In Fig. 10 we show the overlay between the radio brightness at $144 \mathrm{MHz}$ at $20^{\prime \prime}$ and the temperature (first column), the entropy (second column), and the pressure (third column). Temperature and entropy decrease going from the south of the cluster towards the center, and increase again moving further to the north. The radio emission mainly sits in the north of the cluster starting from the location where temperature and entropy increase again. A low entropy strip with values $<80$ (arbitrary units) cuts the cluster in two parts, one hosting bright radio emission and one with very little radio emission. This strip has the same spatial location as the filament F2 and as part of F1. The pressure image is characterized by an elongation in the SSW-NNE, following the merger axis, with larger pressure values along the filament F3. Moreover, an enhancement in the temperature, entropy and pressure images can be identified corresponding to patch $\mathrm{P}$, indicating that this region is dynamically active and therefore favours a possible interpretation as a phoenix source or a revived fossil plasma.

Overall, the bulk of the radio emission is perpendicular to the main elongation in temperature, entropy and pressure, even if a minor elongation of these three thermodynamic quantities can be identified in the same direction of the brightest regions in radio. Moreover, a hint of a pressure gradient seems to be present at the location of the tail of source S1. Available data and the current analysis are not suitable to discriminate whether this could, or could not, be linked to the diffuse emission on large scales observed in this system. Further analysis is left to follow-up works.

\subsection{Spectral index versus temperature and entropy}

Mergers can boost the X-ray luminosity and temperature of a cluster (Ricker \& Sarazin 2001; ZuHone 2011). If a fraction of the gravitational energy dissipated during the merger is available to re-accelerate radio emitting particles, high temperature and entropy regions may be expected to have a flatter spectrum. The study of any correlation between thermodynamic X-ray quantities and spectral index of diffuse radio emission in galaxy clusters has been conducted only for a limited number of clusters and present results are controversial. An anti-correlation between spectral index and temperature is indicated by the results reported by Feretti et al. (2012), who analyzed clusters with $T<8 \mathrm{keV}, 8<T<10 \mathrm{keV}$ and $T>10 \mathrm{keV}$ and derived a trend of decreasing average spectral index for the three samples. A detailed investigation reveals this effect in the cluster A2744 in regions of very different temperatures, spanning from about $5 \mathrm{keV}$ up to more than $10 \mathrm{keV}$ (Orrú et al. 2007). However, Pearce et al. (2017) in A2744 and Shimwell et al. (2014) in the 1E 0657-55.8 cluster do not find significant evidence of this link. Finally, a possible evidence of anticorrelation between spectral index and pseudo-entropy in A2255 has been found by Botteon et al. (2020). In order to better understand the presence of a possible anti-correlation between spectral index and thermodynamic quantities in galaxy clusters, we need to investigate a larger statistical sample.

In Fig. 11, we plot temperature (panel (a)) and pseudo-entropy (panel (b)) against the spectral index. In these plots, the spectral index and the thermodynamic quantities have been averaged in the same regions shown in Fig. 6 in Cova et al. (2019). These plots do not show any clear anti-correlation. The Pearson and Spearman correlation coefficients are $r_{\mathrm{P}}=-0.28$ and $r_{\mathrm{S}}=-0.30$, and $r_{\mathrm{P}}=-0.26$ and $r_{\mathrm{S}}=-0.33$, respectively for the two plots. Therefore, if any relation is present, it is likely very weak.

The lack of any link may be due to the fact that entropy is a tracer not only of merger shocks, but also of physical processes occurring in the ICM, as heating/cooling processes. Moreover, in this cluster, the temperature and the entropy show relatively small variations, with values between 3 and $5 \mathrm{keV}$ and 50 and 150 (arbitrary units), compared e.g. to A2744 and A2255, therefore the lack of 

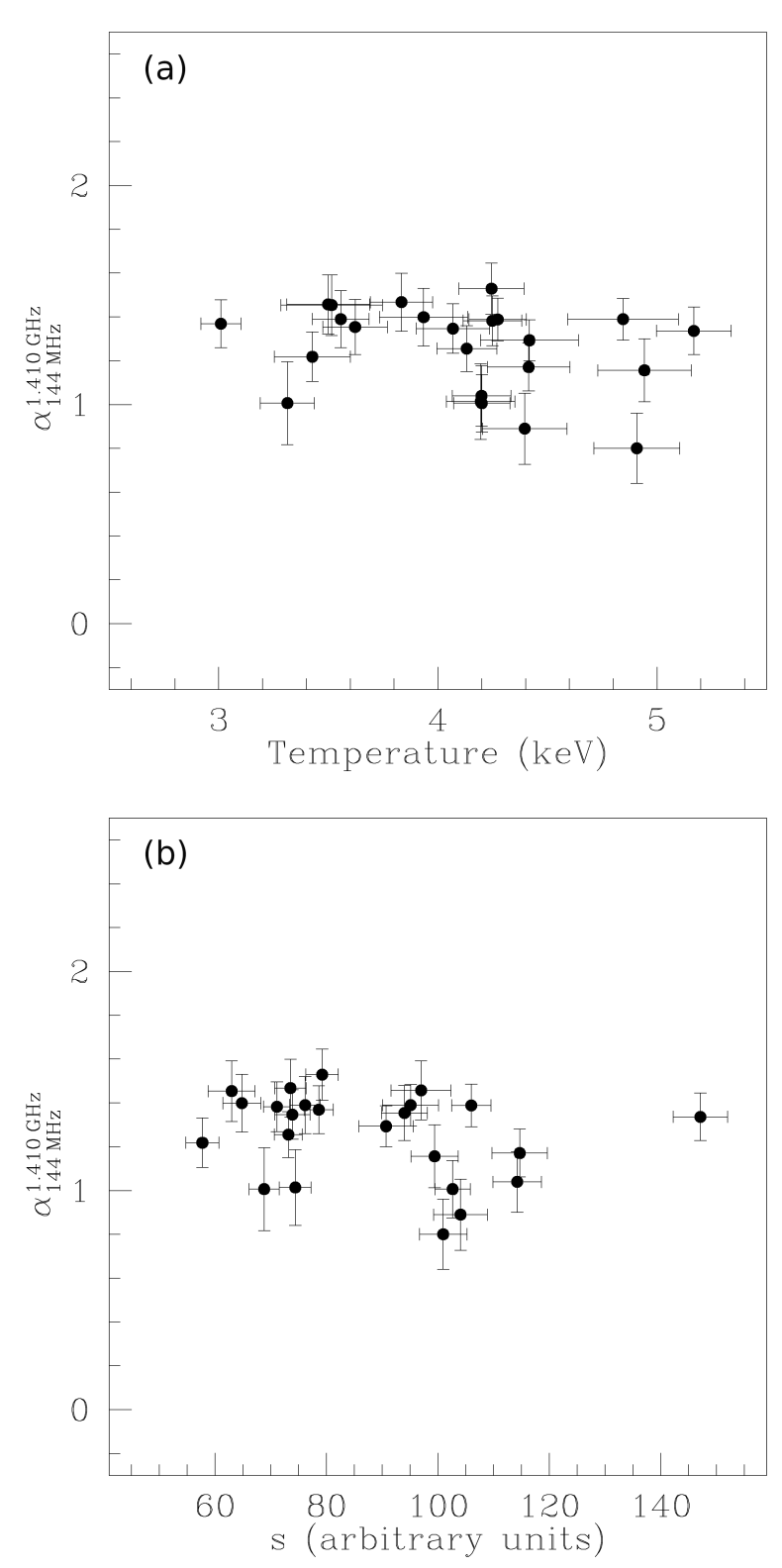

Figure 11. Panel (a): Spectral index between $1.410 \mathrm{GHz}$ and $144 \mathrm{MHz}$ versus thermal gas temperature of the cluster. Panel (b): Spectral index between $1.410 \mathrm{GHz}$ and $144 \mathrm{MHz}$ versus thermal gas pseudo-entropy of the cluster. These plots have been obtained with the low-resolution spectral index images averaged in the same regions of the thermodynamic maps. The temperature and pseudo-entropy values are from Cova et al. (2019).

relations between spectral index and temperature and entropy is not inconsistent with previous findings.

\section{DISCUSSION}

Historically the most prominent diffuse source in A523 has been classified as a radio halo (Giovannini et al. 2011). Subsequent observations revealed some peculiarities (Girardi et al. 2016; Cova et al. 2019):

(i) the radio peak of the source is displaced with respect to the $\mathrm{X}$-ray peak of the system; (ii) the source shows filaments of polarized emission up to 15$20 \%$;

(iii) the radio emission at $1.4 \mathrm{GHz}$ and $144 \mathrm{MHz}$ shows a weak local correlation with the X-rays in the energy range $0.5-2.5 \mathrm{keV}$;

(iv) the radio emission at $1.4 \mathrm{GHz}$ does not follow the correlation with the global luminosity $L_{\mathrm{X}, 0.1-2.4 \mathrm{keV}}$ observed for other radio halos;

(v) the radio emission at $1.4 \mathrm{GHz}$ and $144 \mathrm{MHz}$ does not follow the correlation with the mass $M_{500}$ observed for other radio halos.

In the following we discuss three possible scenarios for the origin of this radio source in light of our results.

Scenario 1. The diffuse emission in A523 could be due to turbulence associated with the complex dynamical state of the system caused by the main and a possible secondary merger. The local radio$\mathrm{X}$-ray comparison at $144 \mathrm{MHz}$ suggests that the primary merger powers diffuse radio emission along the SSW-NNE direction, as shown by the LOFAR observations, while the secondary merger further energizes particles along ESE-WNW, characterized by higher radio brightness than emission in the north-east and in the south-east and clearly detected both at $144 \mathrm{MHz}$ and at $1.4 \mathrm{GHz}$.

Scenario 2. The observed radio emission could be the revival of fossil plasma seeded by the central AGN S1 and later re-accelerated by the merger. In this case the correlations observed for radio halos are not expected. LOFAR images reveal several examples of systems where the AGN plasma has spread cosmic ray electrons over large areas (several hundreds of $\mathrm{kpc}$ ), as recently observed by Brienza et al. (2021), especially in presence of merger turbulence and shocks (e.g., Mandal et al. 2020). However, revived fossil plasma sources and phoenixes typically have a size of $300-400 \mathrm{kpc}$ and steep spectral indices ( $\alpha \gtrsim 1.5$, see Mandal et al. 2020 as well as van Weeren et al. 2019 for a review), different from what is observed in A523.

Scenario 3. The lack of an overall radio-X correlation could suggest that radio and the thermal plasma do not occupy the same volume, with the radio emission being a relic seen in projection, as proposed first by van Weeren et al. (2011). A small inclination of the merging axis with respect to the line of sight $\left(\lesssim 10-20^{\circ}\right)$ could explain why a shock wave and a spectral gradient have been not observed. In cosmological simulations, face-on relics show complex morphologies that consist of filaments, possibly polarized, similar to the diffuse source in A523 (e.g., Skillman et al. 2013; Wittor et al. 2019; Wittor 2021). In these simulations, the spectral indices vary across the relics' surfaces, but they lack the typical spectral steepening towards the cluster center. However, this scenario is not supported by redshift data since there is no evidence for a merger along the line of sight (Girardi et al. 2016; Golovich et al. 2019). Alternatively, an undetected component along the line of sight could be present if the merger is at the turnaround point or if the possible secondary merger is not entirely in the plane of the sky.

\section{CONCLUSIONS}

In this paper we studied the properties of the diffuse emission in A523 by using new LOFAR observations at $144 \mathrm{MHz}$ and new VLA data at 1.410 and $1.782 \mathrm{GHz}$. Our finding can be summarized as follows.

The new radio data reveal an unprecedented amount of detail about the properties of the source at radio wavelengths. The emission at $144 \mathrm{MHz}$ appears more extended than at $1.4 \mathrm{GHz}$, with a total flux density $S_{144 \mathrm{MHz}}=(1.52 \pm 0.31) \mathrm{Jy}$ and a size of about $15^{\prime}$ (i.e., $1.8 \mathrm{Mpc}$ ). The source is characterized by a complex morphology consisting of three bright filaments, two in the north of the system already known from previous observations at $1.4 \mathrm{GHz}$, and a third 
one developing from the bright northern structure to the south of the cluster. The brightest region of the emission is elongated in the ESE-WNW direction also at $144 \mathrm{MHz}$. Thanks to the new LOFAR data, we detect for the first time additional regions of faint emission along SSW-NNE and a bright diffuse synchrotron patch in the south, characterized by a steep spectral index and of unclear origin. A local comparison of the radio and X-ray signal suggests the presence of two components, one brighter in X-ray and less bright in radio and the second the other way around. The new LOFAR and VLA data permit, for the first time, an investigation of the spectral properties of the source. Globally, we derive an average spectral index $\alpha_{144 \mathrm{MHz}}^{1.410 \mathrm{GHz}}=1.2 \pm 0.1$ with a spectral steepening moving towards higher frequency. The spectral index does not show radial steepening but rather a complex spatial distribution.

Overall, our findings suggest that we are observing the overlapping of different structures, powered by the turbulence associated with the primary and a possible secondary merger. Our results and the current optical data do not support a relic interpretation of the source as a whole, while the relic nature of the northern filaments as well as the revived fossil plasma scenario can not be excluded. Although we have derived valuable information about this complex source from the new radio data, additional optical and X-rays observations are necessary to better understand the geometry of the merger and to verify or exclude the presence of possible components along the line of sight.

\section{ACKNOWLEDGEMENTS}

This paper is in memory of our shiny meteor Francesco and of Luciano. We thank the referee whose comments and suggestions helped to strongly improve the presentation of our results. This paper is based (in part) on data obtained with the International LOFAR Telescope (ILT) under project code LC10_024. LOFAR (van Haarlem et al. 2013) is the Low Frequency Array designed and constructed by ASTRON. It has observing, data processing, and data storage facilities in several countries, that are owned by various parties (each with their own funding sources), and that are collectively operated by the ILT foundation under a joint scientific policy. The ILT resources have benefitted from the following recent major funding sources: CNRS-INSU, Observatoire de Paris and Université d'Orléans, France; BMBF, MIWF-NRW, MPG, Germany; Science Foundation Ireland (SFI), Department of Business, Enterprise and Innovation (DBEI), Ireland; NWO, The Netherlands; The Science and Technology Facilities Council, UK; Ministry of Science and Higher Education, Poland. The present project is carried out within the framework of the LOFAR Magnetism Key Science Project https://lof ar-mksp. org/. We acknowledge the computing centre of INAF - Osservatorio Astrofisico di Catania, under the coordination of the WG-DATI of LOFAR-IT project, for the availability of computing resources and support. VV, MM and GB acknowledge support from INAF mainstream project "Galaxy Clusters Science with LOFAR" 1.05.01.86.05. FL acknowledges financial support from the Italian Minister for Research and Education (MIUR), project FARE, project code R16PR59747, project name "FORNAXB". FL acknowledges financial support from the Italian Ministry of University and Research - Project Proposal CIR01_00010. RJvW acknowledges support from the ERC Starting Grant ClusterWeb 804208. AB acknowledges support from the VIDI research programme with project number 639.042.729, which is financed by the Netherlands Organisation for Scientific Research (NWO). AB and DNH acknowledges support from the ERC through the grant
ERC-Stg DRANOEL n. 714245. MB acknowledges support from the Deutsche Forschungsgemeinschaft under Germany's Excellence Strategy - EXC 2121 "Quantum Universe" - 390833306. D.W. is funded by the Deutsche Forschungsgemeinschaft (DFG, German Research Foundation) - 441694982.

\section{DATA AVAILABILITY}

The data underlying this article will be shared on reasonable request to the corresponding author.

\section{REFERENCES}

Akamatsu H., Inoue S., Sato T., Matsusita K., Ishisaki Y., Sarazin C. L., 2013, PASJ, 65,89

Bagchi J., Durret F., Neto G. B. L., Paul S., 2006, Science, 314, 791

Blandford R. D., Ostriker J. P., 1978, ApJ, 221, L29

Bonafede A., et al., 2009, A\&A, 503, 707

Botteon A., et al., 2020, ApJ, 897, 93

Brienza M., et al., 2017, A\&A, 606, A98

Brienza M., et al., 2021, Nature Astronomy,

Brown S., Rudnick L., 2011, MNRAS, 412, 2

Brown S., Duesterhoeft J., Rudnick L., 2011, ApJ, 727, L25

Brunetti G., Jones T. W., 2014, International Journal of Modern Physics D, 23, 1430007

Cassano R., Ettori S., Giacintucci S., Brunetti G., Markevitch M., Venturi T., Gitti M., 2010, ApJ, 721, L82

Cassano R., et al., 2013, ApJ, 777, 141

Clarke T. E., Ensslin T. A., 2006, AJ, 131, 2900

Condon J. J., Cotton W. D., Greisen E. W., Yin Q. F., Perley R. A., Taylor G. B., Broderick J. J., 1998, AJ, 115, 1693

Cova F., et al., 2019, A\&A, 628, A83

Cuciti V., et al., 2021, A\&A, 647, A51

Deiss B. M., Reich W., Lesch H., Wielebinski R., 1997, A\&A, 321, 55

Di Gennaro G., et al., 2018, ApJ, 865, 24

Drury L. O., 1983, Reports on Progress in Physics, 46, 973

Eckert D., Jauzac M., Vazza F., Owers M. S., Kneib J. P., Tchernin C., Intema H., Knowles K., 2016, MNRAS, 461, 1302

Einasto M., Einasto J., Tago E., Müller V., Andernach H., 2001, AJ, 122, 2222

Ensslin T. A., Biermann P. L., Klein U., Kohle S., 1998, A\&A, 332, 395

Erler J., Basu K., Trasatti M., Klein U., Bertoldi F., 2015, MNRAS, 447, 2497

Feretti L., Orrù E., Brunetti G., Giovannini G., Kassim N., Setti G., 2004, A\&A, 423, 111

Feretti L., Giovannini G., Govoni F., Murgia M., 2012, A\&ARv, 20, 54

Giacintucci S., et al., 2005, A\&A, 440, 867

Giovannini G., Feretti L., Venturi T., Kim K. T., Kronberg P. P., 1993, ApJ, 406, 399

Giovannini G., Bonafede A., Feretti L., Govoni F., Murgia M., Ferrari F., Monti G., 2009, A\&A, 507, 1257

Giovannini G., Feretti L., Girardi M., Govoni F., Murgia M., Vacca V., Bagchi J., 2011, A\&A, 530, L5

Girardi M., et al., 2016, MNRAS, 456, 2829

Golovich N., et al., 2019, ApJ, 882, 69

Govoni F., Enßlin T. A., Feretti L., Giovannini G., 2001a, A\&A, 369, 441

Govoni F., Feretti L., Giovannini G., Böhringer H., Reiprich T. H., Murgia M., 2001b, A\&A, 376, 803

Govoni F., Murgia M., Feretti L., Giovannini G., Dallacasa D., Taylor G. B., 2005, A\&A, 430, L5

Govoni F., Murgia M., Xu H., Li H., Norman M. L., Feretti L., Giovannini G., Vacca V., 2013, A\&A, 554, A102

HI4PI Collaboration et al., 2016, A\&A, 594, A116

Hales S. E. G., Baldwin J. E., Warner P. J., 1988, MNRAS, 234, 919

Hales S. E. G., Masson C. R., Warner P. J., Baldwin J. E., 1990, MNRAS, 246, 256 
Hardcastle M. J., et al., 2021, A\&A, 648, A10

Hoang D. N., et al., 2019, A\&A, 622, A20

Hodgson T., Johnston-Hollitt M., McKinley B., Vernstrom T., Vacca V., 2020, arXiv e-prints, p. arXiv:2007.10578

Hurley-Walker N., 2017, arXiv e-prints, p. arXiv:1703.06635

Intema H. T., Jagannathan P., Mooley K. P., Frail D. A., 2017, A\&A, 598, A78

Kale R., Dwarakanath K. S., 2010, ApJ, 718, 939

Kang H., Ryu D., Jones T. W., 2012, ApJ, 756, 97

Large M. I., Mathewson D. S., Haslam C. G. T., 1959, Nature, 183, 1663

Liang H., Hunstead R. W., Birkinshaw M., Andreani P., 2000, ApJ, 544, 686

Lindner R. R., et al., 2014, ApJ, 786, 49

Loi F., et al., 2019, MNRAS, 490, 4841

Mandal S., et al., 2020, A\&A, 634, A4

Markevitch M., Govoni F., Brunetti G., Jerius D., 2005, ApJ, 627, 733

Murgia M., 1996, PhD thesis, University of Bologna

Myers Jerome L.; Well A. D., 2003, printdisabled; librarygenesis,Library Genesis, p. 508

Nagai D., 2006, ApJ, 650, 538

Nagai D., Vikhlinin A., Kravtsov A. V., 2007, ApJ, 655, 98

Norris R. P., et al., 2021, Publ. Astron. Soc. Australia, 38, e003

Offringa A. R., McKinley B., Hurley-Walker et al., 2014, MNRAS, 444, 606

Orrú E., Murgia M., Feretti L., Govoni F., Brunetti G., Giovannini G., Girardi M., Setti G., 2007, A\&A, 467, 943

Pacholczyk A. G., 1970, Radio astrophysics. Nonthermal processes in galactic and extragalactic sources, Series of Books in Astronomy and Astrophysics.. Freeman, San Francisco

Parekh V., Dwarakanath K. S., Kale R., Intema H., 2017, MNRAS, 464, 2752

Pearce C. J. J., et al., 2017, ApJ, 845, 81

Pearson K., 1895, Proceedings of the Royal Society of London Series I, 58, 240

Perley R. A., Butler B. J., 2013, ApJS, 204, 19

Perley R. A., Butler B. J., 2017, ApJS, 230, 7

Pizzo R. F., de Bruyn A. G., Bernardi G., Brentjens M. A., 2011, A\&A, 525, A104

Planck Collaboration et al., 2013, A\&A, 554, A140

Planck Collaboration et al., 2020, A\&A, 641, A6

Rajpurohit K., et al., 2018, ApJ, 852, 65

Rajpurohit K., et al., 2020, A\&A, 642, L13

Rajpurohit K., et al., 2021a, A\&A, 646, A56

Rajpurohit K., et al., 2021b, A\&A, 646, A135

Ricker P. M., Sarazin C. L., 2001, ApJ, 561, 621

Rossetti M., Ghizzardi S., Molendi S., Finoguenov A., 2007, A\&A, 463, 839

Rottgering H. J. A., Wieringa M. H., Hunstead R. W., Ekers R. D., 1997, MNRAS, 290, 577

Schneider P., 2015, Extragalactic Astronomy and Cosmology: An Introduction. Springer-Verlag, Berlin

Shi X., Nagai D., Aung H., Wetzel A., 2020, MNRAS, 495, 784

Shimwell T. W., Brown S., Feain I. J., Feretti L., Gaensler B. M., Lage C., 2014, MNRAS, 440, 2901

Shimwell T. W., et al., 2019, A\&A, 622, A1

Skillman S. W., Xu H., Hallman E. J., O’Shea B. W., Burns J. O., Li H., Collins D. C., Norman M. L., 2013, ApJ, 765, 21

Smirnov O. M., Tasse C., 2015, MNRAS, 449, 2668

Spearman C., 1904, American Journal of Psychology 15, p. 72-101

Tasse C., 2014a, arXiv e-prints, p. arXiv:1410.8706

Tasse C., 2014b, A\&A, 566, A127

Tasse C., et al., 2018, A\&A, 611, A87

Tasse C., et al., 2021, A\&A, 648, A1

Thierbach M., Klein U., Wielebinski R., 2003, A\&A, 397, 53

Urdampilleta I., Akamatsu H., Mernier F., Kaastra J. S., de Plaa J., Ohashi T., Ishisaki Y., Kawahara H., 2018, A\&A, 618, A74

Vacca V., Murgia M., Govoni F., Feretti L., Giovannini G., Orrù E., Bonafede A., 2010, A\&A, 514, A71

Vacca V., Feretti L., Giovannini G., Govoni F., Murgia M., Perley R. A., Clarke T. E., 2014, A\&A, 561, A52

Vacca V., Murgia M., Govoni F., Enßlin T., Oppermann N., Feretti L., Giovannini G., Loi F., 2018a, Galaxies, 6, 142
Vacca V., et al., 2018b, MNRAS, 479, 776

Voit G. M., Kay S. T., Bryan G. L., 2005, MNRAS, 364, 909

Wittor D., 2021, New Astron., 85, 101550

Wittor D., Hoeft M., Vazza F., Brüggen M., Domínguez-Fernández P., 2019, MNRAS, 490, 3987

Xie C., et al., 2020, A\&A, 636, A3

Yu L., Nelson K., Nagai D., 2015, ApJ, 807, 12

Yuan Z. S., Han J. L., Wen Z. L., 2015, ApJ, 813, 77

Zhang X., et al., 2020, A\&A, 642, L3

ZuHone J. A., 2011, ApJ, 728, 54

de Gasperin F., Intema H. T., van Weeren R. J., Dawson W. A., Golovich N., Wittman D., Bonafede A., Brüggen M., 2015, MNRAS, 453, 3483

de Gasperin F., Intema H. T., Frail D. A., 2018, MNRAS, 474, 5008

de Gasperin F., et al., 2019, A\&A, 622, A5

van Weeren R. J., Brüggen M., Röttgering H. J. A., Hoeft M., Nuza S. E., Intema H. T., 2011, A\&A, 533, A35

van Weeren R. J., et al., 2016a, ApJS, 223, 2

van Weeren R. J., et al., 2016b, ApJ, 818, 204

van Weeren R. J., de Gasperin F., Akamatsu H., Brüggen M., Feretti L., Kang

H., Stroe A., Zandanel F., 2019, Space Sci. Rev., 215, 16

van Weeren R. J., et al., 2021, A\&A, 651, A115

This paper has been typeset from a $\mathrm{T}_{\mathrm{E}} \mathrm{X} / \mathrm{LT}_{\mathrm{E}} \mathrm{X}$ file prepared by the author. 